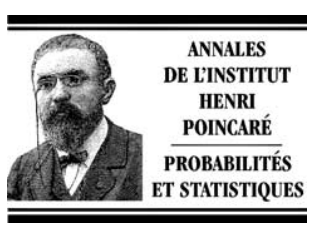

www.elsevier.com/locate/anihpb

\title{
An infinite dimensional central limit theorem for correlated martingales
}

\author{
Ilie Grigorescu ${ }^{1}$ \\ Department of Mathematics, University of Miami, 1365 Memorial Drive, Ungar Building, Room 525, Coral Gables, FL 33146, USA
}

Received 6 March 2003; accepted 27 April 2003

\begin{abstract}
The paper derives a functional central limit theorem for the empirical distributions of a system of strongly correlated continuous martingales at the level of the full trajectory space. We provide a general class of functionals for which the weak convergence to a centered Gaussian random field takes place. An explicit formula for the covariance is established and a characterization of the limit is given in terms of an inductive system of SPDEs. We also show a density theorem for a Sobolevtype class of functionals on the space of continuous functions.
\end{abstract}

(c) 2003 Elsevier SAS. All rights reserved.

\section{Résumé}

L'article présent dérive d'un théorème limite centrale fonctionnelle au niveau de l'espace de toutes les trajectoires continues pour les distributions empiriques d'un système de martingales fortement corrélées. Nous fournissons une classe générale de fonctions pour lesquelles est établie la convergence faible vers un champ aléatoire gaussien centré. Une formule explicite pour la covariance est determinée et on offre une charactérisation de la limite à l'aide d'un système inductif d'équations aux dérivées partielles stochastiques. On démontre également que l'espace de fonctions pour lesquelles le champ des fluctuations converge est dense dans une classe de fonctionnelles de type Sobolev sur l'espace des trajectoires continues.

(c) 2003 Elsevier SAS. All rights reserved.

MSC: primary 60F17; secondary 60G15, 60K35

Keywords: Gaussian random field; Fluctuations from hydrodynamic limit; Central limit theorem

\footnotetext{
E-mail address: igrigore@math.miami.edu (I. Grigorescu).

${ }^{1}$ Research supported in part by McMaster University and The Fields Institute, Ontario, Canada, the University of Utah and the University of Miami.

0246-0203/\$ - see front matter (C) 2003 Elsevier SAS. All rights reserved.

doi:10.1016/j.anihpb.2003.03.001
} 


\section{Introduction}

The classical law of large numbers for empirical measures states that, given a sequence of independent random variables $Z_{1}, Z_{2}, \ldots$ with values in a Polish space $E$ and common distribution $\alpha(d x) \in M(E)$, the random measures $N^{-1} \sum_{i=1}^{N} \delta_{Z_{i}}$ converge weakly in probability to $\alpha(d x)$ as $N \rightarrow \infty$. Furthermore, the fluctuation random field $\xi^{N}=N^{-1 / 2} \sum_{i=1}^{N}\left(\delta_{Z_{i}}-\alpha(d x)\right)$ converges to a centered Gaussian $\xi$ in the sense that, for any test function $g \in C_{b}(E)$, the space of bounded continuous functions on $E$,

$$
\left(g, \frac{1}{\sqrt{N}} \sum_{i=1}^{N}\left(\delta_{Z_{i}}-\alpha(d x)\right)\right)
$$

converges in distribution to a normal random variable with mean zero and variance $\sigma_{\xi}^{2}(g, g)=\operatorname{Cov}_{\alpha}(g, g)$.

We are interested in deriving a central limit theorem when $E$ is the path space $\Omega=C([0, T], \mathbb{R})$ up to time $T>0$ and the random variables $Z_{1}, Z_{2}, \ldots$ are replaced with correlated processes. More precisely, the random field (1.1) can be calculated for the random variables $Z_{i}=z_{i}^{N}(\cdot)$, designating the trajectory of a Markovian system of coupled particles at times $t \in[0, T]$, which can be viewed as path valued random variables. A first step is to extend the notion of fluctuation random field to functionals of the path $\omega(\cdot) \in C([0, T], \mathbb{R})$ up to the time horizon $T>0$ of the particles, that is, at a minimum, to functionals depending of finitely many time marginals $G(\omega(\cdot))=g\left(\omega\left(t_{0}\right), \omega\left(t_{1}\right), \ldots, \omega\left(t_{m}\right)\right)$ for some positive integer $m \geqslant 1$ and $g$ smooth.

The central limit theorem for empirical measures is well known for independent random variables and has been studied as a distribution valued continuous process for the case of Brownian motions by Itô in [9]. In [7], Holley and Stroock introduce the theory of generalized Ornstein-Uhlenbeck processes and prove the central limit theorems for various interacting particle systems. Other limits concerning fluctuation random fields from the hydrodynamic profile of interacting particle systems can be found in [4] for zero range processes in equilibrium, a non-equilibrium result for symmetric simple exclusion appears in [14] and [2] solves the problem for Ginzburg-Landau lattice models. In all these results the limiting random field is a time-indexed continuous Markovian process with values in the space of tempered distributions, that is, can be obtained for the special case $m=0$ and $g \in C^{\infty}(\mathbb{R})$ of Schwartz class.

The result which appears to be the closest in spirit to the present work is [16]. The limit is an infinite dimensional random field, but the coefficients individually satisfy a one-dimensional central limit theorem, a feature due to the mean field character of the model.

Because of the strong correlations (2.8) we cannot keep the bounded continuous functions on $\Omega$ as index set. A natural answer is to use the class of functions with bounded smooth derivatives $C_{b}^{1}(\Omega)$ given in Definition 1 . A price paid for the generalization is that we adopt an example of correlated continuous martingales described by (2.8) in order to have access to concrete calculations. However, this example provides an additional benefit with the derivation of an explicit formula for the covariance function (2.12). The paper has an important constructive component since Sections 2, 3 and the imbedding Theorem 5 are laying the ground for an infinite dimensional result in a general setting. Here they are used in establishing the main result, Theorem 3.

The immediate motivation of the present work is the scaling limit for the Brownian motions with local interaction on the unit circle $S^{1}$ from [5]. After calculating explicitly the asymptotic law of a single particle in the random environment provided by the rest of the interacting system (the tagged particle problem) and establishing that finite subfamilies of particles become independent in the scaling limit (propagation of chaos) one has an immediate weak law of large numbers for the empirical measures associated to the process at the level of the full trajectory space $C\left([0, T], S^{1}\right)$. The process has a product uniform invariant measure and the actual law of the tagged particle process in that case is the Wiener measure on the unit circle with uniform initial distribution. A natural question is whether one can determine the limit for the fluctuation field from the mean, at least in equilibrium.

There is another reason why we need a result at the level of the full path space. Due to the symmetry of interacting diffusions in [5], the hydrodynamic limit and the fluctuation random field from the weak solution to the 
heat equation describing the trajectory of the empirical measures indexed by time are the same as in the case of independent Brownian motions. The quantities present are, from our viewpoint, just one-dimensional marginals of the objects we are interested in. The interaction surfaces only at the level of the path space, or when correlations between configurations observed at consecutive times are taken into account (the history of the process). In a different formulation, the interaction becomes apparent when we consider a multi-color version of the process, as coloring is a weak form of tagging (see, in that sense, the comments in [6] and the approach used in [12] and [13] for symmetric simple exclusion).

The proof of this particle model offers a hint into the nature of the scaling needed for the fluctuation field. Through a path transformation, the system of particles can be converted into a family of martingales on the Skorohod space, adapted to the filtration of the original process. Since the correlations are of the order of the inverse of the number of particles, they survive in the limit. This provides the correct scaling limit (which is also the natural one from the classic CLT).

Unfortunately, even though the path transformation has a smooth asymptotic value which is invertible pathwise, it is discontinuous before the limit and the error from the continuum limit is once again of the order of the square root of the number of particles, a finite but still too large a perturbation in order to establish a CLT like Theorem 3. For our present purposes, one can summarize the example of the induced martingales with the following construction.

Let $\lambda$ and $\bar{\rho}$ be two positive constants. For every $N \in \mathbb{Z}_{+}$we consider a set of $N^{2}$ independent Brownian motions on a probability space $(\mathfrak{W}, \mathcal{F}, P)$, adapted to a filtration $\left\{\mathcal{F}_{t}\right\}_{t} \geqslant 0$, as follows. We shall have, for $1 \leqslant i \leqslant N$ a collection $\beta_{i}(\cdot)$, plus another family of $N(N-1)$ independent Brownian motions $w_{i j}(\cdot)$, with $1 \leqslant i \neq j \leqslant N$ such that $\left\{\beta_{i}(0)\right\}_{1 \leqslant i \leqslant N}$ are i.i.d. with common probability distribution $\mu(d x)$ and $w_{i j}(0)=0 P$-almost surely. Let

$$
z_{i}^{N}(t)=\beta_{i}(t)+\left(\frac{\bar{\rho}}{\lambda+\bar{\rho}}\right) \frac{1}{N} \sum_{j \neq i}\left(\beta_{j}(t)-\beta_{i}(t)\right)+\left(\frac{\sqrt{\lambda \bar{\rho}}}{\lambda+\bar{\rho}}\right) \frac{1}{\sqrt{2 N}} \sum_{k \neq i}\left(w_{i k}(t)-w_{k i}(t)\right) .
$$

The quadratic variation is

$$
\left\langle z_{i}^{N}, z_{i}^{N}\right\rangle(t)=\frac{\lambda}{\lambda+\bar{\rho}} t+\mathrm{O}\left(\frac{1}{N}\right) t
$$

and the cross variation processes for $i \neq j$ are

$$
\left\langle z_{i}^{N}, z_{j}^{N}\right\rangle(t)=\frac{1}{N}\left(\frac{\bar{\rho}}{\lambda+\bar{\rho}}\right) t+\mathrm{O}\left(\frac{1}{N^{2}}\right) t .
$$

It is clear that the construction satisfies assumptions (2.13) and (2.14). The martingales generated in the original problem emulate the interacting diffusions very well and in equilibrium they have identical limit of the empirical measures (in the sense of Theorem 1). In the context of [5], the parameter $\bar{\rho}$ represents the average density of the particles on the unit circle and $\lambda$ controls the intensity of the interaction.

The present paper determines the fluctuation limit in the case of correlated Brownian motions like in the example from above. The discussion following the main results Theorems 2 and 3 and especially Remark 2 after Theorem 3 are helpful in completing the present discussion.

Naturally under weak conditions (Assumption 1), a finite-dimensional CLT will hold, that is, for smooth cylinder test functions. It is remarkable that the covariance can be given in a closed explicit formula (2.12). This fact can be generalized if the limiting one-particle process $Q$ has a time-only dependent generator, but cannot be done along the same lines in the presence of path or spatial coordinate dependence. The passage from cylinder functions to a convenient larger space needs much more stringent conditions (Assumption 2), as it can be seen from Section 5.

Even though the correlated martingales are far from the complexities occurring in interacting particle system, one hopes that, at least in equilibrium, formula (2.12) will be the same. The function space $C_{b}^{1}(\Omega)$ introduced in Definition 1 is a natural candidate for test functions if only we look at the one-dimensional case, for instance. Also, the inductive construction presented in Sections 3 and 4 can be adapted to essentially any Markovian model. 
We give an outline of the way the paper is organized. Section 2 introduces the spaces of cylinder functions, in particular the special cylinder functions of exponential or Schwartz type $\mathcal{E}(\Omega)$, respectively $\mathcal{S}(\Omega)$, in Definition 3 . The Banach space $\mathcal{H}(\Omega, Q)$ presented in Definition 6 allows us to link the space of cylinder functions and the space of test functions $C_{b}^{1}(\Omega)$ (Definition 1) over which Theorem 3 (the infinite dimensional case) is established through an imbedding result - Theorem 5, Section 5. In addition, a few examples of relevant test functions are provided in the remarks after Definition 6 and further down in Proposition 2.

Section 3 lays out an inductive characterization of the random fields through Theorem 4, paired with an inductive characterization of the covariance function in Proposition 3.

Propositions 5 and 6 in Section 4 prove Theorem 2, the central limit theorem for cylinder functions in $(\mathcal{S} \cup \mathcal{E})(\Omega)$. In particular, Proposition 5 and 6 show that the one-dimensional marginal of the limiting Gaussian random field $\xi$ is a time-continuous distribution-valued Markov process solving an Ornstein-Uhlenbeck SPDE, which is consistent with [7] and [9]. Because $\mathcal{S}(\Omega)$ is a linear space we obtain that, for any linear combination $\sum_{l} c_{l} G_{l}$ of functions $G_{l} \in \mathcal{S}(\Omega)$, the random variables $\sum_{l} c_{l}\left(G_{l}, \xi^{N}\right)$ converge to a Gaussian $\left(\sum_{l} c_{l} G_{l}, \xi\right)$. The actual covariance of the limit is obtained after matching the inductive characterization with the actual solution, which is done in Proposition 4. This identification offers an example of a nontrivial solution of the inductive SPDE associated to the half Laplacian and a specific bilinear form $\breve{q}(\cdot, \cdot)$ in the sense of (3.4) in Definition 14.

Section 5 is based on Theorem 5 proved in Appendix A and the asymptotic uniform bound (5.7) from Proposition 9. The latter needs (2.13)-(2.14) from Assumption 2 in order to complete a martingale representation (2.15) through the series of Lemmas $1,2$.

Finally, Appendix A proves non-probabilistic results generally valid in function spaces as well as Theorem 1 which is a trivial hydrodynamic limit in this context.

\section{Definition and results}

Let $\Omega=C([0, T], X)$ be the space of continuous paths up to time $T>0$ on $X$ which will be either the real line $\mathbb{R}$ or the unit circle $S^{1}$. The uniform norm on $X$ will be denoted by $\|\cdot\|$.

Definition 1. Let $\mathbb{C}$ be the set of complex numbers and let $G \in C(\Omega)=C(\Omega, \mathbb{C})$ be the space of complex valued continuous functions and $\omega \in \Omega$ be fixed. Assume there exists a continuous linear mapping $\eta \rightarrow \nabla_{\eta} G(\omega)$, for all $\eta \in \Omega$, and a function $c(G, h)$ depending only on $G$ and $h$ such that, for any $\eta \in \Omega$ and $h \in \mathbb{R}$

$$
\left|G(\omega+h \eta)-G(\omega)-h \nabla_{\eta} G(\omega)\right| \leqslant c(G, h)\|\eta\|^{2}
$$

with $\lim _{h \rightarrow 0}(c(G, h) / h)=0$. The Fréchet derivative will have the strong norm

$$
\|\nabla \cdot G(\omega)\|=\sup _{\eta \neq 0} \frac{\left|\nabla_{\eta} G(\omega)\right|}{\|\eta\|} .
$$

We shall say that $G \in C_{b}^{1}(\Omega)$ if $G(\omega), \nabla . G(\omega)$ are uniformly bounded on $\Omega$. The space $C_{b}^{1}(\Omega)$ is a normed linear space with the norm

$$
\|G\|_{C_{b}^{1}}=\sup _{\omega \in \Omega}(|G(\omega)|+\|\nabla \cdot G(\omega)\|) .
$$

The mappings $\nabla . G(\omega)$ are signed measures on $[0, T]$ depending on $\omega$ and the norm defined by (2.2) is the total variation norm.

Definition 2. We shall denote by $\mathcal{E}(\mathbb{R})$ the set all exponential functions of the form $x \rightarrow \mathrm{e}^{\mathrm{i} \alpha x}$, with $\alpha \in \mathbb{R}$, by $\mathcal{C}(\mathbb{R})$ the set of bounded infinitely differentiable functions on $\mathbb{R}$ with bounded derivatives and by $\mathcal{S}(\mathbb{R})$ the $\operatorname{Sch} w a r t z$ class of functions rapidly decreasing at infinity. 
Definition 3. The functional $G \in C_{b}^{1}(\Omega)$ on the path space $\Omega$ will be said to be $\mathcal{C}$-class cylinder function on $\Omega$ (respectively of $\mathcal{S}$-class or $\mathcal{E}$-class) if there exist a positive integer $m$, an increasing sequence of times $0 \leqslant t_{1}<t_{2}<\cdots<t_{m}$ and a family of functions $g_{l}(x) \in \mathcal{C}(\mathbb{R})$ (respectively of $\mathcal{S}$-class or $\mathcal{E}$-class), $1 \leqslant l \leqslant m$ such that $G(\omega)=\prod_{l=1}^{m} g_{l}\left(\omega\left(t_{l}\right)\right)$. The space of such functions will be denoted by $\mathcal{C}_{\text {cyl }}(\Omega)$ and the linear span of such functions will be denoted by $\mathcal{C}(\Omega)$. In the same way, the space $(\mathcal{E} \cup \mathcal{S})(\Omega)$ is the linear span of cylinder functions with factors belonging either to $\mathcal{E}(\Omega)$ or $\mathcal{S}(\Omega)$.

For $G \in \mathcal{C}_{\text {cyl }}(\Omega)$ we define $a(\omega(\cdot))=\prod_{l=1}^{m-1} g_{l}\left(\omega\left(t_{l}\right)\right)$ and $t^{\prime}=t_{m-1} \geqslant 0$. We denote $\mathcal{M}_{s}=\sigma[\omega(u): 0 \leqslant u \leqslant s]$ the $\sigma$-algebra generated by the continuous paths up to time $s \in[0, T]$. Then $a(\omega(\cdot))$ is a $\mathcal{M}_{t^{\prime}}$-measurable functional on $\Omega$. We shall look at the test function $a(\omega(\cdot)) g(\omega(t))$ for $t \geqslant t^{\prime}$ which we shall call the associate marginal process of $G$ starting at $t=t^{\prime}$. We denote by

$$
\begin{aligned}
& G(\omega, t)=a(\omega(\cdot)) g(\omega(t)), \quad t \geqslant t^{\prime}=t_{m-1}, \\
& \partial G(\omega, t)=a(\omega(\cdot)) g^{\prime}(\omega(t)) \quad \text { and } \quad \partial^{2} G(\omega, t)=a(\omega(\cdot)) g^{\prime \prime}(\omega(t))
\end{aligned}
$$

the (inductive) cylindrical decomposition of $G$ and its derivatives. In the same time, any $G \in \mathcal{C}(\Omega)$ can be written as the sum of functions from $\mathcal{C}_{\text {cyl }}(\Omega)$ with the same $m>0$ by considering the union of all times $t$ from all the terms in $G$ and formally factoring in some constant functions (provided that $t_{m}$ is indeed the largest time present in $G$ ). This allows us to extend the definitions (2.4) to any $G \in \mathcal{C}(\Omega)$.

We consider a probability space $(\mathfrak{W}, \mathcal{F}, P)$, where $\mathcal{F}$ is a filtration $\left\{\mathcal{F}_{t}\right\}_{0 \leqslant t \leqslant T}$ on $\mathfrak{W}$ and take $\sigma^{2}>0$.

Definition 4. We shall denote by $\{Q\}$ the family of laws of the diffusion processes $Q^{v}$ on $\Omega$ with respect to $(\mathfrak{W}, \mathcal{F}, P)$, adapted to an extension of $\left\{\mathcal{F}_{t}\right\}_{t} \geqslant 0$, with generator $\frac{\sigma^{2}}{2} \frac{d^{2}}{d x^{2}}$ and initial distribution $v(d x)$, where $v(d x)$ is a probability measure on $X$. In the following, $Q^{0}$ will designate the Brownian motion with diffusion coefficient $\sigma^{2}$ starting at zero and $Q=Q^{\mu}$ (without superscript) for simplicity.

Definition 5. For $G \in C_{b}^{1}(\Omega)$ we define the linear functional on $\Omega$

$$
\eta \rightarrow\left\langle\nabla_{\eta} G\right\rangle_{Q}=\int_{\Omega} \nabla_{\eta} G(\omega) d Q(\omega) .
$$

Remark. This definition is consistent pointwise since the gradient of $G$ is bounded by $\|\eta\|$. The law $Q$ of the diffusion is the Wiener measure on the path space $\Omega$ and $\omega(\cdot, \mathfrak{w})$ is a random variable measurable with respect to $(\mathfrak{W}, \mathcal{F})$, distributed according to $Q$.

Definition 6. We shall denote by $\mathcal{H}(\Omega, Q)$ the Banach space obtained by completion of the space $C_{b}^{1}(\Omega)$ under the norm $\|\cdot\|_{\mathcal{H}}$ defined as

$$
\|G\|_{\mathcal{H}}^{2}=\int_{\Omega}|G(\omega)|^{2} d Q(\omega)+\int_{\Omega}\|\nabla \cdot G(\omega)\|^{2} d Q(\omega) .
$$

Remark 1. $\mathcal{H}(\Omega, Q)$ is not a Hilbert space. However, for finite-dimensional marginals, that is for cylinder functions $g$ with $m \in \mathbb{Z}_{+}$, we obtain $H^{1}\left(\mathbb{R}^{m+1}\right)$.

Remark 2. Proposition 2 provides a general class of examples of functionals in $\mathcal{H}(\Omega, Q)$ (cylinder functions). Also, an important case of test function $G$ which belongs to $\mathcal{H}(\Omega, Q)$ but not to $\mathcal{S}(\Omega)$ is $G(\omega(\cdot))=\omega(t)$, the projection at a given time $t$. See also the remark after Corollary 1.

Remark 3. If $b(\cdot, \cdot) \in C^{0,2}([0, T], X)$, the functional $G(\omega)=\int_{0}^{t} b(s, \omega(s)) d s$ belongs to $C_{b}^{1}(\Omega)$. 
Proposition 1. The linear functional $\left\langle\nabla_{\eta} G\right\rangle_{Q}$ is square integrable with respect to $Q^{0}$ and

$$
\int_{\Omega}\left|\left\langle\nabla_{\eta} G\right\rangle_{Q}\right|^{2} d Q^{0}(\eta) \leqslant\left(2 \sigma^{2} T\right)\|G\|_{\mathcal{H}}^{2} .
$$

\section{Proof.}

$$
\begin{aligned}
\left|\left\langle\nabla_{\eta} G\right\rangle_{Q}\right|^{2} & =\left|\int_{\Omega}\left(\frac{\nabla_{\eta} G(\omega)}{\|\eta\|}\right)\|\eta\| d Q(\omega)\right|^{2} \\
& \leqslant\left|\int_{\Omega}\|\nabla \cdot G(\omega)\|\|\eta\| d Q(\omega)\right|^{2} \leqslant\left(\int_{\Omega}\|\nabla \cdot G(\omega)\|^{2} d Q(\omega)\right)\|\eta\|^{2} .
\end{aligned}
$$

We can take the expected value with respect to $Q^{0}(\eta)$ and obtain (2.7) using Doob's inequality (see in [15]).

We would like to know how large $\mathcal{H}(\Omega, Q)$ is. The set $\mathcal{S}(\Omega)$ of Schwartz class cylinder functions is a subset of the set of smooth cylinder functions $\mathcal{C}(\Omega)$ which is included in $\mathcal{H}(\Omega, Q)$. Theorem 5 and Proposition 7 will show that $\mathcal{S}(\Omega)$ is dense in $\mathcal{H}(\Omega, Q)$.

Proposition 2. Let $m \in \mathbb{Z}_{+}$and $g\left(x_{0}, x_{1}, \ldots, x_{m}\right)$ be a function in the space $H^{1}\left(\mathbb{R}^{m+1}\right)$, that is with $g$ and its derivative in the sense of distributions square integrable with respect to the Lebesgue measure. If $\mu(d x)$, the initial distribution of $Q$, is absolutely continuous with respect to the Lebesgue measure and the density $\rho(x)$ defined as $\mu(d x)=\rho(x) d x$ is bounded, then $G(\omega)=g\left(\omega\left(t_{0}\right), \ldots, \omega\left(t_{m}\right)\right) \in \mathcal{H}(\Omega, Q)$. Also, if $G$ does not depend on the initial time $t=0$ the statement is valid for arbitrary $\mu(d x)$ as long as $G$ depends on a finite number of times.

Proof. Any function $g \in H^{1}\left(\mathbb{R}^{m+1}\right)$ can be approximated by $g^{S} \in \mathcal{C}\left(\mathbb{R}^{m+1}\right)$ in the $H^{1}$ norm. The statement is proven if we can show it for a Schwartz class function. The preceding lemma has shown that, for any two $\omega, \eta \in \Omega$ we have

$$
\nabla_{\eta} G(\omega)=\sum_{i=0}^{m}\left(\partial_{x_{i}} g\left(\omega\left(t_{0}\right), \ldots, \omega\left(t_{m}\right)\right)\right) \eta\left(t_{i}\right) .
$$

We see that

$$
\begin{aligned}
\left|\nabla_{\eta} G(\omega)\right| & \leqslant\left(\sum_{i=0}^{m}\left(\partial_{x_{i}} g\left(\omega\left(t_{0}\right), \ldots, \omega\left(t_{m}\right)\right)\right)^{2}\right)^{1 / 2}\left(\sum_{i=0}^{m}\left(\eta\left(t_{i}\right)\right)^{2}\right)^{1 / 2} \\
& \leqslant(m+1)^{1 / 2}\left\|\nabla_{\bar{x}} g\left(\omega\left(t_{0}\right), \ldots, \omega\left(t_{m}\right)\right)\right\|_{\mathbb{R}^{m+1}}\|\eta\| .
\end{aligned}
$$

The conditions from the proposition make the joint probability density function of the random variables $\left(\omega\left(t_{0}\right), \ldots, \omega\left(t_{m}\right)\right)$ be a bounded function on $\mathbb{R}^{m+1}$. The formula for the joint density is

$$
\left[\mu\left(y_{0}\right) \prod_{i=1}^{m} p\left(t_{i}-t_{i-1}, y_{i}-y_{i-1}\right)\right] d y_{0} d y_{1} \cdots d y_{m}
$$

with $p(t, y)$ the kernel of the heat equation $u_{t}=\left(\sigma^{2} / 2\right) u_{x x}$. Since the heat equation semigroup produces smooth bounded functions for any $t>0$ irrespective of the initial distribution $\mu(d x)$ the inequality $p\left(t_{i}-t_{i-1}, y_{i}-y_{i-1}\right) \leqslant$ Const $\sigma^{-1}\left(t_{i}-t_{i-1}\right)^{-1 / 2}$ for $i \geqslant 2$ finishes the proof. 
We need to introduce the general setting for Gaussian processes. Let $\mathcal{X}$ be a Banach space with norm $\|\cdot\| \mathcal{X}$. For any complex number $z$ we denote by $\bar{z}$ its complex conjugate.

Definition 7. A continuous bilinear form on $\mathcal{X}$ is a mapping $q: \mathcal{X} \times \mathcal{X} \rightarrow C$ such that, for any $F, G \in \mathcal{X}$, $q(\cdot, G)$ and $\overline{q(F, \cdot)}$ are linear and there exists a constant $c(q)$, independent from $F$ and $G$, such that $|q(F, G)| \leqslant$ $c(q)\|F\|_{\mathcal{X}}\|G\|_{\mathcal{X}}$. We shall write $q \in B(\mathcal{X})$. The bilinear form is called symmetric if $q(F, G)=\overline{q(G, F)}$, nonnegative if $q(G, G) \geqslant 0$ and positive if $q(G, G)>0$ if $G \neq 0$.

A random process indexed by $\mathcal{A}$ is, by definition, a collection of real-valued random variables $\{\xi\}=\left\{\xi_{a}\right\}_{a \in \mathcal{A}}$ on a probability space $(\mathfrak{W}, \mathcal{F}, P)$. The sub-algebra $\Sigma$ generated by

$$
C_{a_{1}, a_{2}, \ldots, a_{n}, B}=\left\{\mathfrak{w} \in \mathfrak{W}:\left(\xi_{a_{1}}(\mathfrak{w}), \ldots, \xi_{a_{n}}(\mathfrak{w})\right) \in B\right\} \in \mathcal{F}
$$

where $n \in \mathbb{Z}_{+}, a_{1}, a_{2}, \ldots, a_{n} \in \mathcal{A}$ and $B \in \mathcal{B}\left(\mathbb{R}^{n}\right)$ are arbitrary, allows us to define a probability measure on $\mathbb{R}^{\mathcal{A}}$ with the $\sigma$-field generated by the finite dimensional projections. For any finite set $\mathcal{A}_{0}=\left\{a_{1}, a_{2}, \ldots, a_{n}\right\} \subseteq \mathcal{A}$ we define the probability measure on $\mathbb{R}^{F}$, called the finite dimensional distribution of $\xi$ on $\mathcal{A}_{0}$ by

$$
\widetilde{P}_{\mathcal{A}_{0}}(B)=P\left(\left(\xi_{a_{1}}(\mathfrak{w}), \ldots, \xi_{a_{n}}(\mathfrak{w})\right) \in B\right) .
$$

The consistency condition of the finite dimensional distributions is respected. For any two finite subsets of $\mathcal{A}$ such that $\mathcal{A}_{0}^{\prime} \subseteq \mathcal{A}_{0}$ we define $\pi_{\mathcal{A}_{0}^{\prime}}$ the projection of $\mathbb{R}^{\mathcal{A}_{0}}$ onto $\mathbb{R}^{\mathcal{A}_{0}^{\prime}}$ and then

$$
\widetilde{P}_{\mathcal{A}_{0}} \circ \pi_{\mathcal{A}_{0}^{\prime}}^{-1}=\widetilde{P}_{\mathcal{A}_{0}^{\prime}}
$$

Kolmogorov's extension theorem shows that under these circumstances there exists a probability measure on $\mathbb{R}^{\mathcal{A}}$ and the $\sigma$-field generated by the finite dimensional cylinder functions denoted by $\widetilde{P}$ such that, if $F$ is a finite subset of $\mathcal{A}$ and $\pi_{\mathcal{A}_{0}}$ is the projection of $\mathbb{R}^{\mathcal{A}}$ onto $\mathbb{R}^{\mathcal{A}_{0}}$, then

$$
\widetilde{P} \circ \pi_{\mathcal{A}_{0}}^{-1}=\widetilde{P}_{\mathcal{A}_{0}}
$$

Definition 8. Let $\xi^{N}=\left\{\xi_{a}^{N}\right\}_{a \in \mathcal{A}}$ defined for all $N>0$ and a separate $\xi=\left\{\xi_{a}\right\}_{a \in \mathcal{A}}$ be random processes indexed by $\mathcal{A}$. We shall say that $\xi^{N}$ converges to $\xi$ as $N \rightarrow \infty$ if the finite dimensional distributions of $\xi^{N}$ converge weakly to the finite dimensional distributions of $\xi$.

Definition 9. The random process $\xi$ indexed by $\mathcal{A}$ is called Gaussian (centered Gaussian) if all linear combinations of $\xi_{a}$ with $a \in \mathcal{A}$ are Gaussian (centered Gaussian).

Definition 10. Let $\mathcal{X}$ be a Banach space. The random process $\xi$ indexed by $F \in \mathcal{X}$ with the property that the mapping $F \rightarrow \xi_{F}$ belongs to $\mathcal{X}^{\prime}$, the space of linear functionals on $\mathcal{X}$, is called a random field on $\mathcal{X}$ and we shall write $\xi_{F}=(F, \xi)$. In case the random process defined this way is Gaussian (centered Gaussian) we shall say that $\xi$ is a Gaussian (centered Gaussian) random field.

For $\mathcal{X}$ equal to the space $\mathcal{H}(\Omega, Q)$ and $q(\cdot, \cdot)$ a nonnegative continuous symmetric bilinear form on $\mathcal{H}(\Omega, Q)$, there exists a centered Gaussian random field $\xi=\xi(\mathfrak{w})$ on $\mathcal{X}$ with covariance $q\left(F-\langle F\rangle_{Q}, G-\langle G\rangle_{Q}\right)$ for any two $F, G \in \mathcal{H}(\Omega, Q)$. Here and in the following $\langle G\rangle_{Q}=\int_{\Omega} G d Q(\omega)$. In this case we shall denote the variance by $\sigma_{\xi}^{2}(\cdot, \cdot)$. 
We are now ready to formulate the assumptions needed for our results. For every positive integer $N$, we consider a family of $N$ continuous square-integrable martingales with respect to $P$ and $\left\{\mathcal{F}_{t}\right\}_{0 \leqslant t \leqslant T}$, taking values in $X$, denoted by $\left\{z_{i}^{N}(\cdot, \mathfrak{w})\right\}_{1 \leqslant i \leqslant N}$. The cross variation processes $\left\{\left\langle z_{i}^{N}, z_{j}^{N}\right\rangle(t, \mathfrak{w})\right\}_{t} \geqslant 0,1 \leqslant i, j \leqslant N$ can be written as

$$
\left\langle z_{i}^{N}, z_{i}^{N}\right\rangle(t, \mathfrak{w})=\int_{0}^{t} \sigma_{N, i}^{2}(s, \mathfrak{w}) d s \quad \text { and } \quad\left\langle z_{i}^{N}, z_{j}^{N}\right\rangle(t, \mathfrak{w})=\int_{0}^{t} k_{N}^{i j}(s, \mathfrak{w}) d s
$$

where $\sigma_{N, i}^{2}(\cdot, \mathfrak{w})$ and $\left|k_{N}^{i j}(\cdot, \mathfrak{w})\right|$ are time-integrable a.s. with respect to $P$.

Equivalently, by the martingale representation theorem, possibly by extending the $\sigma$-field $\mathcal{F}$, one can define the $N$-dimensional Brownian motion $\{\mathbf{z}(\cdot, \mathfrak{w})\}$ by

$$
d z_{i}(t, \mathfrak{w})=\sum_{l=1}^{L} \mathbf{r}_{i l}(t, \mathfrak{w}) d w_{l}(t, \mathfrak{w}), \quad 1 \leqslant i \leqslant N, 1 \leqslant l \leqslant L,
$$

where $L \in \mathbb{Z}_{+}$and $\{\mathbf{w}(\cdot, \mathfrak{w})\}=\left\{w_{l}(\cdot, \mathfrak{w})\right\}_{1 \leqslant l \leqslant L}$ is an $L$-dimensional Brownian motion adapted to $\mathcal{F}$ and $\mathbf{R}(t, \mathfrak{w}) \mathbf{R}^{*}(t, \mathfrak{w})=\left(\sum_{l} \mathbf{r}_{i l}(t, \mathfrak{w}) \mathbf{r}_{l j}(t, \mathfrak{w})\right)_{i j}$ is the correlation matrix of $\{\mathbf{z}(\cdot, \mathfrak{w})\}$ with elements given by the integrands from (2.8).

In order to derive a finite-dimensional central limit theorem for the empirical measures associated to the family of martingales $\left\{z_{i}^{N}(\cdot)\right\}_{1 \leqslant i \leqslant N}$ we shall only need the following condition.

Assumption 1 (Finite dimensional CLT). There exist $\sigma^{2}>0$ and $\gamma>-1$ such that

$$
\lim _{N \rightarrow \infty} E\left[\int_{0}^{T} \sum_{i=1}^{N}\left(\sigma_{N, i}^{2}(s, \mathfrak{w})-\sigma^{2}\right)^{2} d s\right]=0
$$

and

$$
\lim _{N \rightarrow \infty} E\left[\int_{0}^{T} \frac{1}{N^{2}} \sum_{1 \leqslant i \neq j \leqslant N}\left(N k_{N}^{i j}(s, \mathfrak{w})-\sigma^{2} \gamma\right)^{2} d s\right]=0
$$

Theorem 1. Under Assumption 1, if there exists a probability measure $\mu(d x)$ on $X$ such that the initial empirical measures $N^{-1} \sum_{i=1}^{N} \delta_{z_{i}^{N}(0)}$ converge weakly to $\mu(d x)$ in probability, then the empirical measure $N^{-1} \sum_{i=1}^{N} \delta_{z_{i}(\cdot)}$ converges weakly to $Q$ in probability.

The proof of Theorem 1 is in Appendix A.

Theorem 2. Under Assumption 1, where (2.9) and (2.10) are satisfied with $\gamma>-1$ and the initial values of the martingales $z_{1}^{N}(0), \ldots, z_{N}^{N}(0)$ are independent with distribution $\mu(d x)$ on $X$, the random field

$$
\xi^{N}=\frac{1}{\sqrt{N}} \sum_{i=1}^{N}\left(\delta_{z_{i}^{N}(\cdot)}-Q\right)
$$

on $\mathcal{S}_{\text {cyl }}(\Omega)$ converges in the sense of Definition 8 to a centered Gaussian random field $\xi$ on $\mathcal{S}_{\mathrm{cyl}}(\Omega)$ with covariance

$$
\sigma_{\xi}^{2}(F, G)=\int_{\Omega}\left(F(\omega)-\langle F\rangle_{Q}\right) \overline{\left(G(\omega)-\langle G\rangle_{Q}\right)} d Q(\omega)+\gamma \int_{\Omega}\left\langle\nabla_{\eta} F\right\rangle_{Q} \overline{\left\langle\nabla_{\eta} G\right\rangle_{Q}} d Q^{0}(\eta)
$$

for any $F, G \in \mathcal{S}_{\text {cyl }}(\Omega)$. 
A stronger set of condition is adopted for an infinite dimensional central limit theorem.

Assumption 2 (Infinite dimensional CLT). There exist $\sigma^{2}>0$ and $\gamma>-1$, as well as constants $C(\sigma, N)$, $C(\gamma, N)$, for all $N \geqslant 1$ independent from $\mathfrak{w} \in \mathfrak{W}$ and $t \in[0, T]$, with the property $\lim _{N \rightarrow \infty} C(\sigma, N)=0$ and $\lim _{N \rightarrow \infty} C(\gamma, N)=0$ such that properties (2.13) and (2.14) are valid $P$-almost surely:

$$
\sqrt{N} \max _{1 \leqslant i \leqslant N}\left[\sup _{0 \leqslant t \leqslant T}\left|\sigma_{N, i}^{2}(t, \mathfrak{w})-\sigma^{2}\right|\right] \leqslant C(\sigma, N)
$$

and

$$
\max _{1 \leqslant i, j \leqslant N}\left[\sup _{0 \leqslant t \leqslant T}\left|N k_{N}^{i j}(t, \mathfrak{w})-\sigma^{2} \gamma\right|\right] \leqslant C(\gamma, N)
$$

Remark 1. Lemma 1 ensures the existence of a system of martingales with these properties, in other words, that the covariance matrix of the martingales stays positive definite.

Remark 2. Assumption 2 implies Assumption 1.

Remark 3. In the following we shall omit the random element $\mathfrak{w}$ when not necessary.

Theorem 3. Under Assumption 2, where (2.13) and (2.14) are satisfied with $\gamma>-1$ and the initial values of the martingales $z_{1}^{N}(0), \ldots, z_{N}^{N}(0)$ are independent with distribution $\mu(d x)$ on $X$, the random field $(2.11)$ on $C_{b}^{1}(\Omega)$ converges in the sense of Definition 8 to a centered Gaussian random field $\xi$ on $C_{b}^{1}(\Omega)$ with covariance (2.12) for any $F, G \in C_{b}^{1}(\Omega)$. Furthermore, since the covariance (2.12) is continuous with respect to the norm $\|\cdot\|_{\mathcal{H}}$, the limit $\xi$ can be extended to a centered Gaussian random field on $\mathcal{H}(\Omega, Q)$.

Remark 1. The random field $\xi^{N}$ is in fact a random measure on $\Omega$ for any $N>0$. As a consequence, $\xi^{N}$ has values in $(\mathcal{H}(\Omega, Q))^{\prime}$ almost surely. However, one cannot carry out the limit uniformly over $G \in \mathcal{H}(\Omega, Q)$ to prove that the limit $\xi$ has values in $(\mathcal{H}(\Omega, Q))^{\prime}$ even in the independent case. Still, if we drop the requirement that the measure on $(H(\Omega, Q))^{\prime}$ be countably additive (no longer a measure in the proper sense) we can define a so-called cylindrical measure (see [1], Section 3.9).

Remark 2. Suppose we keep the correspondence $\sigma^{2}=\lambda /(\lambda+\bar{\rho})$ and $\gamma=\bar{\rho} / \lambda$, in view of the example (1.2). Then we can see that, in the strong interacting case when $\lambda \rightarrow 0$, the first term of the covariance (2.12), corresponding to the classical central limit theorem (noncorrelated case) will vanish, while the second part, corresponding to the correlation, will tend to one. This can be seen because, as the measure $Q^{N}$ converges to a degenerate measure, the factor $E^{Q^{0}}\left[\left(\left\langle\nabla_{\eta} G\right\rangle_{Q}\right)^{2}\right] \sim E^{Q^{0}}\left[\|\eta\|^{2}\right]$, which is of the order of $\lambda$. Multiplied by $\gamma=\bar{\rho} / \lambda$ we obtain a limit of order one. This is natural when the particles are, in fact, moving deterministically. The remaining randomness originates from the classical CLT for the initial positions of the particles. On the other hand, the case of very rare particles $\bar{\rho} \rightarrow 0$ eliminates correlation. The weak interaction case $\lambda \rightarrow \infty$ pushes the diffusion coefficient to one (independent Brownian motions) and the asymptotic correlation vanishes again.

Theorem 3 extends the finite dimensional result of Theorem 2 to the infinite dimensional space $C_{b}^{1}(\Omega)$. Theorem 5 imbeds the space of cylinder functions into $\mathcal{H}(\Omega, Q)$, providing a density theorem $\overline{\mathcal{S}(\Omega)} \mathcal{H} \supseteq C_{b}^{1}(\Omega)$, where the subscript designates the norm (2.6). In order to make use of this imbedding, we need an uniform bound (also a tightness estimate) with respect to $N$ on the second moments of the random field $\xi^{N}$. This is done through (5.7) from Proposition 9. In order to understand better Assumption 2 we have to write

$$
z_{i}^{N}(t)=y_{i}^{N}(t)+\frac{1}{\sqrt{N}} d_{i}^{N}(t), \quad 1 \leqslant i \leqslant N,
$$


as in Lemma 2, where $\left\{y_{i}^{N}(\cdot)\right\}_{1 \leqslant i \leqslant N}$ are independent Brownian motions and the continuous martingales $\left\{d_{i}^{N}(\cdot)\right\}_{1 \leqslant i \leqslant N}$ have asymptotically bounded moments of some order $r>2$. The construction can be viewed as an orthonormalization procedure. Assumption 2 is sufficient for this construction as well as for a certain simplicity of the result. Following the proof of Proposition 9 and Lemmas 1 and 2 one can try to relax the assumptions (2.13), (2.14) to

$$
E\left[\int_{0}^{T}\left(\sigma_{N, i}^{2}(s, \mathfrak{w})-\sigma^{2}\right)^{2} d s+\sum_{j \neq i} \int_{0}^{T}\left(k_{N}^{i j}(s, \mathfrak{w})-\frac{\sigma^{2} \gamma}{N}\right)^{2} d s\right] \sim \mathrm{o}\left(\frac{1}{N}\right)
$$

uniformly in $1 \leqslant i \leqslant N$. However, this weaker set of conditions complicates the proof of Proposition 8 . We shall not pursue this direction in the present paper.

\section{Inductive characterization of a random field}

The following definitions formulate the main conditions needed for the characterization of a centered Gaussian random field by induction on the maximum number of times $m$ appearing in the test functions from $\mathcal{C}(\Omega)$. We recall that $Q$ is a Brownian motion as in Definition 4 .

Definition 11. Let $q(\cdot, \cdot) \in B\left(C_{b}^{1}(\Omega)\right)$ be symmetric, nonnegative and continuous in the $\mathcal{H}(\Omega, Q)$ norm as in Definition 7. For any pair of functionals $F, G \in C_{b}^{1}(\Omega)$ of the form

$$
F(\omega(\cdot))=f(\omega(0)) \quad \text { and } \quad G(\omega(\cdot))=g(\omega(0))
$$

with $f, g \in C_{b}^{1}(X)$, the bilinear form $q_{0}(f, g)=q(F, G)$ is well defined, symmetric, nonnegative and continuous with respect to the induced norm $\|\cdot\|_{\mathcal{H}(X, \mu)}$ on $\mathcal{H}(X, \mu)$. This one-dimensional bilinear form will be called the one-dimensional marginal of $q(\cdot, \cdot)$ at $t=0$.

Definition 12. A bilinear form $u(\cdot, \cdot) \in B\left(C_{b}^{1}(\Omega)\right)$ is said translation invariant if $u(F+c, G)=u(F, G)$ and $u(F, G+c)=u(F, G)$ for any $c \in C$.

Definition 13. Let $\check{q}(\cdot, \cdot) \in B\left(C_{b}^{1}(\Omega)\right)$ be a positive symmetric bilinear form continuous with respect to the $\mathcal{H}(\Omega, Q)$ norm. We shall say that $u(\cdot, \cdot)$ solves inductively the differential equation for the operator $\left(\sigma^{2} / 2\right) \Delta$ and the bilinear form $\check{q}(\cdot, \cdot)$ with initial marginal at time $t=0$ denoted by $q_{0}(\cdot, \cdot)$ if

$$
\begin{aligned}
& u(F(\omega, t), G(\omega, t))-u\left(F\left(\omega, t^{\prime}\right), G\left(\omega, t^{\prime}\right)\right) \\
& \quad=\frac{\sigma^{2}}{2} \int_{t^{\prime}}^{t}\left(u\left(\partial^{2} F(\omega, s), G(\omega, s)\right)+u\left(F(\omega, s), \partial^{2} G(\omega, s)\right)+2 \breve{q}(\partial F(\omega, s), \partial G(\omega, s))\right) d s
\end{aligned}
$$

for all $F, G \in \mathcal{E}(\Omega)$ and $u_{0}(F, G)=q_{0}(F, G)$ as in Definition 11 .

Proposition 3. Let $\check{q}(\cdot, \cdot) \in B\left(C_{b}^{1}(\Omega)\right)$ be a positive continuous symmetric bilinear form and $q_{0}(\cdot, \cdot)$ is its one-dimensional marginal. If there exists a translation invariant bilinear form $u(\cdot, \cdot)$ on $C_{b}^{1}(\Omega)$ starting at $q_{0}\left(f-\langle f\rangle_{\mu}, g-\langle g\rangle_{\mu}\right)$ in the sense of Definition 11 satisfying the inductive equation (3.2), then $u(\cdot, \cdot)$ is unique.

Remark 1. The inductive partial differential equation is valid for all smooth cylinder functions. What Proposition 3 and later Theorem 4 imply is that it is enough to verify it for the $\mathcal{E}$-class cylinder functions. Given that $q(\cdot, \cdot)$ is continuous in the $\|\cdot\|_{\mathcal{H}}$ norm, we can go from $\mathcal{E}(\Omega)$ to $\mathcal{S}(\Omega)$ and finally to $\mathcal{H}(\Omega, Q)$. 
Remark 2. Proposition 3 is valid if we assume Eq. (3.2) is verified for pairs $(F, G)$ with $F=G$.

Proof of Proposition 3. The difference $v(\cdot, \cdot)$ of two solutions of (3.2) is a translation invariant bilinear form starting at zero and is a solution of the same Eq. (3.2) where the term in $\breve{q}(\cdot, \cdot)$ is cancelled, namely a solution to

$$
\begin{aligned}
& v(F(\omega, t), G(\omega, t))-v\left(F\left(\omega, t^{\prime}\right), G\left(\omega, t^{\prime}\right)\right) \\
& \quad=\frac{\sigma^{2}}{2} \int_{t^{\prime}}^{t}\left(v\left(\partial^{2} F(\omega, s), G(\omega, s)\right)+v\left(F(\omega, s), \partial^{2} G(\omega, s)\right)\right) d s .
\end{aligned}
$$

We want to show that such a solution is zero. To prove this fact, we concentrate on purely cylindrical functions and proceed by induction on the number of factors present. For all $G \in \mathcal{S}(\Omega)$ one can write $G(\omega(\cdot))=\sum_{l} G_{l}(\omega(\cdot))$, where we made the assumption that the summation runs through a finite set, with $G_{l} \in \mathcal{S}_{\text {cyl }}(\Omega)$. Any function $G_{l}$ can be written, according to (2.4), in the form $G_{l}(\omega, t)=a_{G, l}(\omega(\cdot)) g_{G, l}(\omega(t))$ where $t \geqslant t^{\prime}$ is the largest time variable present in $G$ and $t^{\prime}$ is the next time smaller than $t$. This implies that

$$
G(\omega, t)=\int_{\mathbb{R}} \sum_{l}\left[\left(a_{G, l}(\omega) \exp (\mathrm{i} \alpha \omega(t))\right) \hat{g}_{G, l}(\alpha)\right] d \alpha,
$$

for $\alpha \in \mathbb{R}$ and the analogous formulas are valid for $F \in \mathcal{S}(\Omega)$. Then, we replace $F(\omega, t)$ by $\int_{\mathbb{R}} a_{F}(\omega) \mathrm{e}^{\mathrm{i} \alpha \omega(t)} \hat{g}_{F}(\alpha) d \alpha$ and $G(\omega, t)$ by $\int_{\mathbb{R}} a_{G}(\tilde{\alpha}) \mathrm{e}^{\mathrm{i} \tilde{\alpha} \omega(t)} \hat{g}_{G}(\tilde{\alpha}) d \tilde{\alpha}$ where $(\alpha, \tilde{\alpha}) \in \mathbb{R}^{2}$.

We first prove the uniqueness in the case of $F_{\alpha}(\omega, t)=a_{F}(\omega) \mathrm{e}^{\mathrm{i} \alpha \omega(t)}$ and $G_{\tilde{\alpha}}(\omega, t)=a_{G}(\omega) \mathrm{e}^{\mathrm{i} \tilde{\alpha} \omega(t)}$. We look at (3.3) as an ordinary differential equation with unknown $q\left(F_{\alpha}(\omega, t), G_{\tilde{\alpha}}(\omega, t)\right)$. We can calculate explicitly the solution which is unique and equal to zero as long as the induction hypothesis on the values at time $t=t^{\prime}$ is satisfied. The solutions will be bounded and hence we can write the full formula in terms of $F_{\alpha}(\omega, t)=a_{F}(\omega) \mathrm{e}^{\mathrm{i} \alpha \omega(t)}$ and $G_{\tilde{\alpha}}(\omega, t)=a_{G}(\omega) \mathrm{e}^{\mathrm{i} \tilde{\alpha} \omega(t)}$ as

$$
v(F(\omega, t), G(\omega, t))=\int_{\mathbb{R}} \int_{\mathbb{R}} v\left(F_{\alpha}(\omega, t), G_{\tilde{\alpha}}(\omega, t)\right) \hat{g}_{F}(\alpha) \hat{g}_{G}(\tilde{\alpha}) d \alpha d \tilde{\alpha}
$$

by using the bilinearity of $v(\cdot, \cdot)$ and passing to the limit in the Riemann integral over $\mathbb{R}^{2}$, which proves our assertion. The extension to all $F, G \in \mathcal{S}(\Omega)$ is granted by linearity. Theorem 5 and Proposition 7 show us that $\mathcal{S}(\Omega)$ is dense in $\mathcal{H}(\Omega, Q)$ in the $\|\cdot\|_{\mathcal{H}}$ norm. This is enough to extend the result to $\mathcal{H}(\Omega, Q)$, under the $\|\cdot\|_{\mathcal{H}}$ norm since we know that the unique solution to (3.3) is continuous in the $\|\cdot\|_{\mathcal{H}}$ norm. This concludes the proof.

Definition 14. Let $\breve{q}(\cdot, \cdot) \in B\left(C_{b}^{1}(\Omega)\right)$ a nonnegative symmetric continuous bilinear form on $C_{b}^{1}(\Omega)$ and $\xi(\mathfrak{w})$ a random field on $C_{b}^{1}(\Omega)$, measurable with respect to $(\mathfrak{W}, \mathcal{F}, P)$. Assume that for any $G \in \mathcal{C}_{\text {cyl }}(\Omega)$ there exists a standard Brownian motion $\{\beta(t, \mathfrak{w})\}_{t \geqslant 0}$ adapted to the filtration $\left\{\mathcal{F}_{t}\right\}_{t \geqslant 0}$ such that, if $G(\omega(\cdot))$ is written $G(\omega, t)=$ $a(\omega(\cdot)) g(\omega(t))$ as in $(2.4)$, the process $\{(G(\omega, t), \xi(\mathfrak{w}))\}_{t \geqslant t^{\prime}}$ starting at $t=t^{\prime}$ from $\left(a(\omega(\cdot)) g\left(\omega\left(t^{\prime}\right)\right), \xi(\mathfrak{w})\right) \in \mathcal{F}_{t^{\prime}}$ (also called the associate marginal process of $G$ ) satisfies the SPDE

$$
d(G(\omega, t), \xi(\mathfrak{w}))=\frac{\sigma^{2}}{2}\left(\partial^{2} G(\omega, t), \xi(\mathfrak{w})\right) d t+\sigma \sqrt{\check{q}(\partial G(\omega, t), \partial G(\omega, t))} d \beta(t, \mathfrak{w}) .
$$

Then we shall say that $\xi$ satisfies the inductive $\operatorname{SPDE}(3.4)$ with correlation $\check{q}(\cdot, \cdot)$ with respect to $Q$.

Remark 1. For any time $t$ the functional of $\omega(\cdot)$ equal to $G(\omega, t)=a(\omega(\cdot)) g(\omega(t))$ belongs to $\mathcal{C}_{\text {cyl }}(\Omega)$ if $a(\cdot), g(\cdot)$ belong to the continuous class (respectively $\mathcal{E}_{\text {cyl }}(\Omega)$ if $a(\cdot), g(\cdot)$ belong to the exponential class) so $(G(\omega, t), \xi(\mathfrak{w}))$ is well defined. 
Remark 2. For proving our main result we only need $\check{q}(\cdot, \cdot)$ defined in (4.2). However, Theorem 4 is true for a general $C_{b}^{1}(\Omega)$-valued bilinear form $\check{q}(\cdot, \cdot)$.

Theorem 4. Let $\breve{q}(\cdot, \cdot) \in B\left(C_{b}^{1}(\Omega)\right)$ be a positive symmetric bilinear form continuous with respect to the $\mathcal{H}(\Omega, Q)$ norm. Assume also that $q(\cdot, \cdot) \in B\left(C_{b}^{1}(\Omega)\right)$, a positive symmetric bilinear form continuous with respect to the $\mathcal{H}(\Omega, Q)$ norm, solves inductively $E q .(3.2)$ for $\breve{q}(\cdot, \cdot)$ with initial marginal $q_{0}(\cdot, \cdot)$ as in Definition 13 . If $Z=Z(\mathfrak{w})$ is a random field on $C_{b}^{1}(\Omega)$, measurable with respect to $\mathcal{F}$, such that

(i) the one-dimensional restriction $Z_{0}$ of $Z$ at time $t=0$ is a centered Gaussian with covariance $q_{0}\left(f-\langle f\rangle_{\mu}, g-\right.$ $\left.\langle g\rangle_{\mu}\right)$ and

(ii) $Z$ satisfies the inductive $S P D E(3.4)$ for all $G \in \mathcal{E}(\Omega)$,

then $Z$ can be uniquely extended to a centered Gaussian random field on $\mathcal{H}(\Omega, Q)$ with covariance

$$
\sigma_{Z}^{2}(F, G)=q\left(F-\langle F\rangle_{Q}, G-\langle G\rangle_{Q}\right)
$$

for any two $F, G \in \mathcal{H}(\Omega, Q)$.

Remark 1. The condition that $Z$ be a random field on $C_{b}^{1}(\Omega)$ is weaker than $Z$ be indexed by the Banach space $\mathcal{H}(\Omega, Q)$. However, the iterated SPDE, Theorem 5 and the fact that $q(\cdot, \cdot)$ can be extended to $B(\mathcal{H}(\Omega, Q))$ will show that $Z$ is a random field on $\mathcal{H}(\Omega, Q)$.

Remark 2. This is a uniqueness theorem only. For the covariance prescribed in our problem (2.11)-(2.12) the existence is proven by direct verification of the conditions of Theorem 4 applied to $q(\cdot, \cdot)$ from $(2.12)$ with $\breve{q}(\cdot, \cdot)$ given in (4.2). We note that in the proof of Proposition 4 the bilinear form $\check{q}(\cdot, \cdot)$ is split in two components (4.3) and (4.4) corresponding to the "independent" and "coupled" parts of the covariance.

Proof of Theorem 4. Let's denote the value of $(G(\omega, t), Z(\mathfrak{w}))$ by $Z(G(\omega, t))$ for cylinder functions $G \in$ $(\mathcal{E} \cup \mathcal{S})(\Omega)$. In general, we shall suppress $\mathfrak{w}$ in the following. We shall apply the Ito formula to the function $\phi\left(Z(G(\omega, t))\right.$, with $\phi(x)=x^{2}$ and obtain, after taking the expected value, that

$$
d E\left[Z(G(\omega, t))^{2}\right]=\left(\sigma^{2} E\left[Z(G(\omega, t)) Z\left(\partial^{2} G(\omega, t)\right)\right]+\sigma \check{q}(\partial G(\omega, t), \partial G(\omega, t))\right) d t .
$$

This proves that $E[Z(G(\omega, t)) Z(F(\omega, t))]$ satisfies (3.2) by polarization. Next, the uniqueness argument of Proposition 3 implies that

$$
E[Z(F(\omega, t)) Z(G(\omega, t))]=q(F-\langle F\rangle, G-\langle G\rangle) \leqslant C\|F\|_{\mathcal{H}}\|G\|_{\mathcal{H}}
$$

We need to show that $Z(G(\omega, t))$ is a centered Gaussian. For all $G \in \mathcal{S}(\Omega)$ one can write $G(\omega(\cdot))=$ $\sum_{l} G_{l}(\omega(\cdot))$, where we made the assumption that the summation runs through a finite set, with $G_{l} \in \mathcal{S}_{\text {cyl }}(\Omega)$. This implies that $G(\omega, t)=\int_{\mathbb{R}} \sum_{l}\left[\left(a_{l}(\omega) \exp (\mathrm{i} \alpha \omega(t))\right) \hat{g}_{l}(\alpha)\right] d \alpha$ exactly as detailed in the proof of Proposition 3 . Then, Eq. (3.4) gives the SPDE satisfied by the process $\{Z(G(\omega, t))\}_{t \geqslant t^{\prime}}$ starting at $t=t^{\prime}$ from $Z\left(a(\omega(\cdot)) g\left(\omega\left(t^{\prime}\right)\right)\right)$ (also called the associate marginal process of $G$ )

$$
d Z(G(\omega, t))=\frac{\sigma^{2}}{2} Z\left(\partial^{2} G(\omega, t)\right) d t+\sigma \sqrt{\check{q}(\partial G(\omega, t), \partial G(\omega, t))} d \beta(t) .
$$

The inductive SPDE (3.4) for a function of type $a_{l}(\omega) \exp (\mathrm{i} \alpha \omega(t))$ reduces to a classical Ornstein-Uhlenbeck process. We know by the induction hypothesis that at start $t=t^{\prime}$ the process was a centered Gaussian. Let's denote by $Z\left(G_{\alpha, l}(\omega, t)\right)$ the solution to (3.4) for $G_{\alpha, l}(\omega, t)=a_{l}(\omega) \exp (\mathrm{i} \alpha \omega(t))$. The first two terms of the equation are linear. The quadratic form $q(\cdot, \cdot)$ has the property

$$
q\left(\partial G_{\alpha, l}(\omega, t), \partial G_{\alpha, l}(\omega, t)\right)=|\alpha|^{2} \check{q}\left(G_{\alpha, l}(\omega, t), G_{\alpha, l}(\omega, t)\right) .
$$


We derive that

$$
Z\left(G_{\alpha, l}(\omega, t)\right)-Z\left(G_{\alpha, l}\left(\omega, t^{\prime}\right)\right)+\frac{\alpha^{2} \sigma^{2}}{2} \int_{t^{\prime}}^{t} Z\left(G_{\alpha, l}(\omega, s)\right) d s-|\alpha| \sigma \int_{t^{\prime}}^{t} \sqrt{\check{q}\left(G_{\alpha, l}(s), G_{\alpha, l}(s)\right)} d \beta(s)=0 .
$$

The initial $Z\left(G\left(\omega, t^{\prime}\right)\right)$ is a mean zero normal random variable by the induction hypothesis. We integrate the Ornstein-Uhlenbeck SDE $t \rightarrow Z(G(\omega, t))$ and obtain

$$
\begin{aligned}
Z(G(\omega, t))= & \int_{\mathbb{R}} \sum_{l} Z\left(G_{\alpha, l}(\omega, t)\right) \hat{g}_{l}(\alpha) d \alpha \\
= & \int_{\mathbb{R}} \sum_{l} Z\left(G_{\alpha, l}\left(\omega, t^{\prime}\right)\right) \exp \left(-\frac{\alpha^{2} \sigma^{2}}{2}\left(t-t^{\prime}\right)\right) \hat{g}_{l}(\alpha) d \alpha \\
& +\sigma \int_{t^{\prime}}^{t} \int_{\mathbb{R}} \sum_{l} \exp \left(-\frac{\alpha^{2} \sigma^{2}}{2}(t-s)\right)\left(\sqrt{\check{q}\left(G_{\alpha, l}(s), G_{\alpha, l}(s)\right)}\right)|\alpha| \hat{g}_{l}(\alpha) d \alpha d \beta(s) .
\end{aligned}
$$

We need to justify the integration along the real line of the solutions $Z\left(G_{\alpha, l}(\omega, t)\right)$. Let $G \in \mathcal{C}_{\text {cyl }}(\Omega), G(\omega, t)=$ $a(\omega) g(\omega(t))$ such that $a(\omega) \in \mathcal{C}(\Omega), g \in \mathcal{S}(X)$ and, for any $r \in \mathbb{Z}_{+}$, let

$$
R_{r}(G(\omega, t))=a(\omega) \sum_{-r^{2} \leqslant k \leqslant r^{2}} \mathrm{e}^{\mathrm{i} \alpha_{k} \omega(t)} \hat{g}\left(\alpha_{k}\right)\left(\alpha_{k+1}-\alpha_{k}\right)
$$

be the Riemann sum for the partition $\Delta_{r}$ obtained by dividing the interval $[-r, r]$ into $2 r^{2}$ equal subintervals with partition points denoted by $\alpha_{k},-r^{2} \leqslant k \leqslant r^{2}$. Then, $R_{r}(G(\omega, t)) \in \mathcal{C}(\Omega)$ and

$$
\lim _{r \rightarrow \infty}\left\|R_{r}(G(\omega, t))-G(\omega, t)\right\|_{\mathcal{H}}=0
$$

This follows from the properties of the inverse Fourier transform on the real line, since $a(\omega)$ is a bounded smooth functional.

The real integral

$$
G(\omega, t)=\int_{\mathbb{R}}\left(\sum_{l}\left(a_{l}(\omega) \exp (\mathrm{i} \alpha \omega(t))\right) \hat{g}_{l}(\alpha)\right) d \alpha
$$

is the $\|\cdot\|_{\mathcal{H}^{-}}$-limit of Riemannian sums $R_{m}\left(\sum_{l} G_{l}(\omega, t)\right)$ (Eq. (3.7)). Since there exists a constant $C$ such that $E\left[Z(G)^{2}\right] \leqslant C\|G\|_{\mathcal{H}}^{2}$, we can derive that the sequence $\left\{Z\left(\sum_{l} R_{m}\left(G_{l}(\omega, t)\right)\right)\right\}_{m} \geqslant 1$ is tight with limit $Z(G(\omega, t))$. A similar reasoning proves that the last integral involving the bilinear form $\check{q}(\cdot, \cdot)$ can be integrated along $\alpha \in \mathbb{R}$ as a consequence of Plancherel's identity for $g^{\prime}(x)$.

We notice that if

$$
V\left(\omega, t^{\prime}\right)=E\left[\sum_{l} a_{l}(\omega) g_{l}(\omega(t)) \mid \mathcal{F}_{t^{\prime}}\right]
$$

then

$$
\int_{\mathbb{R}} \sum_{l} Z\left(G_{\alpha, l}\left(\omega, t^{\prime}\right)\right) \exp \left(-\frac{\alpha^{2} \sigma^{2}}{2}\left(t-t^{\prime}\right)\right) \hat{g}_{l}(\alpha) d \alpha=2 \pi Z\left(V\left(\omega, t^{\prime}\right)\right)
$$

which implies from the induction hypothesis that the first term in (3.6) is a zero mean Gaussian measurable with respect to $\mathcal{F}_{t^{\prime}} . V\left(\omega, t^{\prime}\right)$ is indeed in $\mathcal{S}(\Omega)$ as the summation of convolutions of functions in $\mathcal{S}(\Omega)$ with the transition 
probability of $Q$ from time $t^{\prime}$ to time $t$. The second term is a stochastic integral of a deterministic function of time depending on $G$ against a Brownian motion on $\left[t^{\prime}, t\right]$, that is a process with independent increments. The sigma fields they are supported on are independent. This concludes the induction step needed to prove that the solution is a mean zero normal random variable.

By piecing together the time marginals present in any $G \in \mathcal{S}(\Omega)$ we have shown that there exists a unique mean zero Gaussian random field satisfying the inductive SPDE (3.4) for any $G \in \mathcal{S}(\Omega)$ provided we start at time $t=0$ with a random field compatible with $q(\cdot, \cdot)$, that is, having the one-dimensional bilinear form equal to the marginal bilinear form of $q\left(F-\langle F\rangle_{\mu}, G-\langle G\rangle_{\mu}\right)$ at $t=0$ (Definition 11). It has been shown in the first part of the proof that the covariance is exactly $q\left(F-\langle G\rangle_{Q}, F-\langle G\rangle_{Q}\right)$. This and the continuity of $q(\cdot, \cdot)$ with respect to the $\mathcal{H}$ norm enables us to extend $Z$ to $\mathcal{H}(\Omega, Q)$, which concludes the proof.

Corollary 1. Let $q(\cdot, \cdot), \breve{q}(\cdot, \cdot)$ and $q_{0}\left(f-\langle f\rangle_{\mu}, g-\langle g\rangle_{\mu}\right)$ be exactly like in Theorem 4 . If there exists a random field $Z$ on $C_{b}^{1}(\Omega)$ such that the restriction $Z_{0}$ of $Z$ to one-dimensional functionals of the type (3.1) is a centered Gaussian with covariance $q_{0}\left(f-\langle f\rangle_{\mu}, g-\langle g\rangle_{\mu}\right)$ and for any $F, G \in \mathcal{E}(\Omega)$ the processes

$$
Z(G(\omega, t))-Z\left(G\left(\omega, t^{\prime}\right)\right)-\frac{\sigma^{2}}{2} \int_{t^{\prime}}^{t} Z\left(\partial^{2} G(\omega, s)\right) d s
$$

and

$$
\begin{aligned}
& Z(F(\omega, t)) Z(G(\omega, t))-Z\left(F\left(\omega, t^{\prime}\right)\right) Z\left(G\left(\omega, t^{\prime}\right)\right) \\
& \quad-\frac{\sigma^{2}}{2} \int_{t^{\prime}}^{t}\left(Z\left(\partial^{2} F(\omega, s)\right) Z(G(\omega, s))+Z(F(\omega, s)) Z\left(\partial^{2} G(\omega, s)\right)+2 \check{q}(\partial F(\omega, s), \partial G(\omega, s))\right) d s
\end{aligned}
$$

are $\left(\mathcal{F}_{t}, P\right)$-martingales, then $Z$ is unique and has covariance $q\left(F-\langle F\rangle_{Q}, G-\langle G\rangle_{Q}\right)$.

Remark. It is easy to see that $G(\omega)=\pi_{t}(\omega)=\omega(t)$ belongs to $\mathcal{H}(\Omega, Q)$ for any time $t \geqslant 0$. The corollary implies that $Z\left(\pi_{t}(\omega)\right)$ is a Brownian motion with respect to the filtration of the process.

\section{The proof for a special class of functions}

The proof of Theorem 2 is the main result of this section.

Proposition 4. The bilinear form $q(\cdot, \cdot) \in B(\mathcal{H}(\Omega, Q))$, defined in $(2.12)$ as

$$
q(F, G)=\int_{\Omega}\left(F(\omega)-\langle F\rangle_{Q}\right) \overline{\left(G(\omega)-\langle G\rangle_{Q}\right)} d Q(\omega)+\gamma \int_{\Omega}\left\langle\nabla_{\eta} F\right\rangle_{Q} \overline{\left\langle\nabla_{\eta} G\right\rangle_{Q}} d Q^{0}(\eta)
$$

is symmetric, nonnegative, translation invariant and satisfies the inductive equation (3.2) starting at $q_{0}(f, g)=$ $\operatorname{Cov}(f, g)$ for any $f, g \in C_{b}^{1}(X)$, where

$$
\operatorname{Cov}(f, g)=\int_{X}\left(f(x)-\langle f\rangle_{\mu}\right)\left(\overline{g(x)-\langle g\rangle_{\mu}}\right) \mu(d x)
$$

with correlation

$$
\check{q}(F, G)=\int_{\Omega} F(\omega) \bar{G}(\omega) d Q(\omega)+\gamma \int_{\Omega}\left\langle\nabla_{\eta} F\right\rangle_{Q} \overline{\left\langle\nabla_{\eta} G\right\rangle_{Q}} d Q^{0}(\eta) .
$$


Proof. Let

$$
q_{1}(F, G)=E^{Q}[(F(\omega)-\langle F\rangle)(\overline{G(\omega)-\langle G\rangle})]
$$

and

$$
q_{2}(F, G)=E^{Q^{0}}\left[\left(\left\langle\nabla_{\eta} F\right\rangle_{Q}\right)\left(\overline{\left\langle\nabla_{\eta} G\right\rangle_{Q}}\right)\right] .
$$

We want to show that both satisfy the inductive equation (3.2) with

$$
\check{q}_{1}(\partial F, \partial G)=E^{Q}[(\partial F(\omega))(\overline{\partial G(\omega)})]
$$

and

$$
\check{q}_{2}(\partial F, \partial G)=\left(E^{Q}[\partial F(\omega)]\right)\left(E^{Q}[\overline{\partial G(\omega)}]\right) .
$$

The equation for $\check{q}_{1}(\partial F, \partial G)$ is obtained as in the case of uncorrelated martingales by Itô formula. We look at $q_{2}(\cdot, \cdot)$ only. Let $G(\omega, t)=a_{G}(\omega) g_{G}(\omega(t))$ and $F(\omega, t)=a_{F}(\omega) g_{F}(\omega(t))$. Then, if $\langle\cdot\rangle_{\omega}$ denotes the expected value with respect to $Q$,

$$
\begin{aligned}
& \left\langle\nabla_{\eta} G\right\rangle_{Q}=\left\langle\nabla_{\eta} a_{G}(\omega) g_{G}(\omega(t))\right\rangle_{\omega}+\left\langle a_{G}(\omega) g_{G}^{\prime}(\omega(t))\right\rangle_{\omega} \eta(t), \\
& \frac{d}{d t}\left\langle\nabla_{\eta} a_{G}(\omega) g_{G}(\omega(t))\right\rangle_{\omega}=\frac{\sigma^{2}}{2}\left\langle\nabla_{\eta} a_{G}(\omega) g_{G}^{\prime \prime}(\omega(t))\right\rangle_{\omega}, \\
& \frac{d}{d t}\left\langle a_{G}(\omega) g_{G}^{\prime}(\omega(t))\right\rangle_{\omega}=\frac{\sigma^{2}}{2}\left\langle a_{G}(\omega) g_{G}^{\prime \prime \prime}(\omega(t))\right\rangle_{\omega} .
\end{aligned}
$$

The analogous formulas hold for $F$. The $d t$ term from the quadratic variation of the Brownian motion $\eta(\cdot)$ is

$$
\sigma^{2}\left(\left\langle a_{F}(\omega) g_{F}^{\prime}(\omega(t))\right\rangle_{\omega}\left\langle\overline{a_{G}(\omega) g_{G}^{\prime}(\omega(t))}\right\rangle_{\omega}\right) d t
$$

equal to $\sigma^{2} \check{q}_{2}(\partial F(\omega, t), \partial G(\omega, t)) d t$. We superpose the two solutions and obtain (3.2) for our random field $\xi$.

Remark. An alternate way to prove the proposition is to consider a pair of i.i.d. Brownian motions $\omega_{1}(\cdot), \omega_{2}(\cdot)$ with law $Q=Q^{\mu}$ and another independent Brownian motion $\eta(\cdot)$ with law $Q^{0}$, write the Itô formula for (4.3), (4.4) (before averaging) for the three-dimensional system, and finally take the expected value.

Proposition 5. For any $G \in(\mathcal{E} \cup \mathcal{S})(\Omega)$, that is such that there exists a positive integer $m$ and a function $g\left(x_{0}, x_{1}, \ldots, x_{m}\right)$ in the linear span of cylinder functions obtained as products of either $\mathcal{S}(\mathbb{R})$ or $\mathcal{E}(\mathbb{R})$ for which $G(\omega)=g\left(\omega\left(t_{0}\right), \ldots, \omega\left(t_{m}\right)\right)$,

$$
\limsup _{N \rightarrow \infty} E\left|\left(G, \xi^{N}\right)\right|^{2} \leqslant c(G)
$$

with $c(G)$ depending only on $g$ and $T$.

Proof. We shall proceed by induction to show (4.7). If $m=1$ and $t_{0}=0$ we have the classical central limit theorem. Assume (4.7) is valid for $G$ depending on at most $m-1$ times. In general we only need to do the proof for cylinder functions since the extension to the general class of functions needed in the proposition is done by linearity and depends, as required, only on $g$ and $T$. To make things precise $g\left(x_{0}, x_{1}, \ldots, x_{m}\right)=\prod_{i=0}^{m} g_{i}\left(x_{i}\right)$ and the constant $c(g, T)$ corresponding to $\prod_{i=0}^{m-1} g_{i}\left(x_{i}\right)$ is denoted by $c(g, m-1, T)$. Also we denote $t_{m-1}$ by $t^{\prime}$. Even though we need $g(x)$ in the Schwartz class, we shall investigate first the case $g(x)=\exp (\mathrm{i} \alpha x)$ where $\mathrm{i}=\sqrt{-1}$. We shall use the notation $G(\omega, t)=G_{\alpha}(\omega, t)$ for such a test function later on in the proof when needed. 
For any $F, G \in \mathcal{C}(\Omega)$ we can write the generalized Itô formula for stochastic integrals with respect to martingales (see [8]) for the product $\left\{\left(F(\omega, t), \xi^{N}\right)\left(\bar{G}(\omega, t), \xi^{N}\right)\right\}_{t \geqslant t^{\prime}}$. We recall that for every finite $N$ the random fields $\xi^{N}$ are finite random measures on the path space $\Omega$ and

$$
\frac{\partial}{\partial t}\langle G(\omega, t)\rangle_{Q}=\frac{\sigma^{2}}{2}\left\langle\partial^{2} G(\omega, t)\right\rangle_{Q}
$$

Then

$$
\begin{aligned}
\left(F(\omega, t), \xi^{N}\right)\left(\bar{G}(\omega, t), \xi^{N}\right)-\left(F\left(\omega, t^{\prime}\right), \xi^{N}\right)\left(\bar{G}\left(\omega, t^{\prime}\right), \xi^{N}\right) & \\
= & \frac{1}{2 N} \int_{t^{\prime}}^{t} \sum_{1 \leqslant i, j \leqslant N}\left[\left(\sigma_{N, i}^{2}(s) \partial^{2} F\left(z_{i}(\cdot), s\right)-\sigma^{2}\left\langle\partial^{2} F\right\rangle_{Q}\right)\left(\bar{G}\left(z_{i}(\cdot), s\right)-\langle\bar{G}\rangle_{Q}\right)\right. \\
& \left.+\left(F\left(z_{i}(\cdot), s\right)-\langle F\rangle_{Q}\right)\left(\sigma_{N, j}^{2}(s) \partial^{2} \bar{G}\left(z_{j}(\cdot), s\right)-\sigma^{2}\left\langle\partial^{2} \bar{G}\right\rangle_{Q}\right)\right] \\
& \times 2 \int_{t^{\prime}}^{t}\left[\frac{1}{N} \sum_{1 \leqslant i \leqslant N}\left(\partial F\left(z_{i}(\cdot), s\right)\right)\left(\partial \bar{G}\left(z_{i}(\cdot), s\right)\right)\left(\sigma_{N, i}^{2}(s)\right)\right] d s \\
& \times\left[\frac{1}{N^{2}} \sum_{1 \leqslant i, j \leqslant N}\left(\partial F\left(z_{i}(\cdot), s\right)\right)\left(\partial \bar{G}\left(z_{j}(\cdot), s\right)\right)\left(N k_{N}^{i j}(s)\right)\right] d s+M_{N}(t),
\end{aligned}
$$

where $M_{N}(t)$ is a martingale. We can re-write the above formula as

$$
\begin{aligned}
& \left(F(\omega, t), \xi^{N}\right)\left(\bar{G}(\omega, t), \xi^{N}\right)-\left(F\left(\omega, t^{\prime}\right), \xi^{N}\right)\left(\bar{G}\left(\omega, t^{\prime}\right), \xi^{N}\right) \\
& =\frac{\sigma^{2}}{2} \frac{1}{N} \int_{t^{\prime}}^{t}\left(\sum _ { 1 \leqslant i , j \leqslant N } \left[\left(\partial^{2} F\left(z_{i}(\cdot), s\right)-\left\langle\partial^{2} F\right\rangle_{Q}\right)\left(\bar{G}\left(z_{j}(\cdot), s\right)-\langle\bar{G}\rangle_{Q}\right)\right.\right. \\
& \left.\quad+\left(\partial^{2} \bar{G}\left(z_{j}(\cdot), s\right)-\left\langle\partial^{2} \bar{G}\right\rangle_{Q}\right)\left(F\left(z_{i}(\cdot), s\right)-\langle F\rangle_{Q}\right)\right] \\
& \left.\quad \times 2\left[\frac{1}{N} \sum_{1 \leqslant i \leqslant N}\left(\partial F\left(z_{i}(\cdot), s\right)\right)\left(\partial \bar{G}\left(z_{i}(\cdot), s\right)\right)\right]\right) d s \\
& \quad+\sigma^{2} \gamma \int_{t^{\prime}}^{t}\left[\frac{1}{N^{2}} \sum_{1 \leqslant i, j \leqslant N}\left(\partial F\left(z_{i}(\cdot), s\right)\right)\left(\partial \bar{G}\left(z_{j}(\cdot), s\right)\right)\right] d s+M_{N}(t)+\mathcal{E}_{N}(t)
\end{aligned}
$$

The error term is

$$
\left|\mathcal{E}_{N}(t)\right| \leqslant C(F, G) \frac{1}{N} \sum_{i=1}^{N} \int_{t^{\prime}}^{t}\left(\sqrt{N}\left|\sigma_{N, i}^{2}(s)-\sigma^{2}\right|\right) d s+\frac{1}{N^{2}} \sum_{1 \leqslant i, j \leqslant N} \int_{t^{\prime}}^{t}\left|N k_{N}^{i, j}(s)-\gamma \sigma^{2}\right| d s,
$$

where $C(F, G)$ is a constant depending on the supremum over $\Omega$ of the functions $F, \partial F, \partial^{2} F$ and the analogue values for $G$. More precisely $\mathcal{E}_{N}(t)=\int_{t^{\prime}}^{t} e_{N}(s) d s$ with

$$
\limsup _{N \rightarrow \infty} E\left[\mathcal{E}^{2}(t)\right] \leqslant T E\left[\int_{0}^{T} e_{N}^{2}(s) d s\right]
$$

by Schwarz inequality for the time integral. The latter bound goes to zero as $N \rightarrow \infty$ from formula (4.9) plus Assumption 1, Eqs. (2.9), (2.10), and once more by Schwarz applied to the average over $N$. 
Let $F=G=a(\omega) g(\omega(t))$ with $g(x)=\exp (\mathrm{i} \alpha x)$. If we denote $u_{N}(t)=E\left|\left(G(\omega, t), \xi^{N}\right)\right|^{2}$ we derive

$$
u_{N}(t)-u_{N}\left(t^{\prime}\right)=-\alpha^{2} \sigma^{2} \int_{t^{\prime}}^{t} u_{N}(s)-q(G(\omega, s), G(\omega, s)) d s+\mathcal{E}_{N}(t)
$$

where we denote, for simplicity,

$$
\begin{aligned}
q(s)= & q(G(\omega, s), G(\omega, s))=\int_{\Omega} G(\omega, s) \bar{G}(\omega, s) d Q(\omega) \\
& +\gamma\left(\int_{\Omega} G(\omega, s) d Q(\omega) \int_{\Omega} \bar{G}(\omega, s) d Q(\omega)\right) \leqslant 2\left(\sup _{\bar{x} \in \mathbb{R}^{m+1}}|g(\bar{x})|\right)^{2} .
\end{aligned}
$$

We differentiate, solve the ODE and obtain

$$
\begin{aligned}
u_{N}(t)= & u_{N}\left(t^{\prime}\right) \exp \left(-\alpha^{2} \sigma^{2}\left(t-t^{\prime}\right)\right)+\alpha^{2} \sigma^{2} \int_{t^{\prime}}^{t} q(s) \exp \left(-\alpha^{2} \sigma^{2}(t-s)\right) d s \\
& +\int_{t^{\prime}}^{t} e_{N}(s) \exp \left(-\alpha^{2} \sigma^{2}(t-s)\right) d s .
\end{aligned}
$$

This shows that

$$
\limsup _{N \rightarrow \infty} u_{N}(t) \leqslant \limsup _{N \rightarrow \infty} u_{N}\left(t^{\prime}\right)+\alpha^{2} \sigma^{2} T c_{m}(g)=c(g, m-1, T)+\alpha^{2} \sigma^{2} T c_{m}(g),
$$

where we denoted $2\left(\sup _{\bar{x} \in \mathbb{R}^{m+1}}|g(\bar{x})|\right)^{2}$ by $c_{m}(g)$.

Since $\lim \sup _{N \rightarrow \infty} u_{N}\left(t^{\prime}\right) \leqslant c(g, m-1, T)$ by the induction hypothesis, relation (4.12) proves the tightness of $\left\{\left(G(\omega, t), \xi^{N}\right)\right\}_{N}$ in the special case $g(x)=\exp (\mathrm{i} \alpha x)$. Let $g(x)$ be a function in the Schwartz space and let $g(x)=\int_{\mathbb{R}} \hat{g}(\alpha) \exp (\mathrm{i} \alpha x) d \alpha$ where $\hat{g}(\alpha) \in \mathcal{S}(C)$ as well. Before passing to the limit as $N \rightarrow \infty$ the random fields $\xi^{N}$ are finite random measures on $\Omega$. The integration over the real line of $\alpha$ can be viewed as the limit of finite Riemann sums converging in the uniform norm on $C_{b}(\Omega)$, since, for a finite $N$, the random fields $\xi^{N}$ are finite measures on $\Omega$. We can write

$$
\left(G(\omega, t), \xi^{N}\right)=\int_{\mathbb{R}}\left(G_{\alpha}(\omega, t), \xi^{N}\right) \hat{g}(\alpha) d \alpha
$$

The sequence will be tight if $\lim _{\sup } \rightarrow \infty\left|\left(G(\omega, t), \xi^{N}\right)\right|^{2}<\infty$. We calculate

$$
E\left|\left(G(\omega, t), \xi^{N}\right)\right|^{2}=E\left|\int_{\mathbb{R}}\left(G_{\alpha}(\omega, t), \xi^{N}\right) \hat{g}(\alpha) d \alpha\right|^{2} \leqslant \int_{\mathbb{R}} E\left|\left(G_{\alpha}(\omega, t), \xi^{N}\right)\right|^{2} \hat{g}^{2}(\alpha) d \alpha .
$$

Then, according to Eq. (4.13),

$$
\limsup _{N \rightarrow \infty} E\left|\left(G(\omega, t), \xi^{N}\right)\right|^{2} \leqslant \int_{\mathbb{R}}\left(c(g, m-1, T)+\alpha^{2} \sigma^{2} T c_{m}(g)\right) \hat{g}^{2}(\alpha) d \alpha \leqslant c(g, m, T) .
$$

The very last step is to consider a general function $G$ which will be a finite sum of cylinder functions. The bound we obtain will depend on the number of terms in $G$, determined exclusively by the test function $g$ and the endpoint $T$ of the time interval. 
Proposition 6. For any pair $F, G \in(\mathcal{E} \cup \mathcal{S})(\Omega)$ as in Proposition 5, the families of processes $\left\{\left(F(\omega, \cdot), \xi^{N}\right)\right\}_{N>0}$ and $\left\{\left(G(\omega, \cdot), \xi^{N}\right)\right\}_{N>0}$ defined for $t \geqslant t^{\prime}$ (as in formula (2.4)) are tight and any pair of limit points, denoted by $Z(F(\omega, \cdot))$ and $Z(G(\omega, \cdot))$ respectively, satisfy the inductive SPDE (3.4) with correlation form (4.2).

Proof. The $m=0$ and $t=0$ case is the classic central limit theorem. At time $t=t^{\prime}$ the functionals $\left(G\left(\omega, t^{\prime}\right), \xi^{N}\right)$ are uniformly square integrable in $N$ either by the induction hypothesis or directly from Proposition 5 . The differences $\left(G(\omega, t), \xi^{N}\right)-\left(G(\omega, s), \xi^{N}\right)$ will be treated analogously with (4.8).

$$
\begin{aligned}
\left(G(\omega, t), \xi^{N}\right)-\left(G(\omega, s), \xi^{N}\right)= & \frac{1}{2 \sqrt{N}} \int_{s}^{t} \sum_{1 \leqslant j \leqslant N}\left(\sigma_{N, j}^{2}(u) \partial^{2} G\left(z_{j}(\cdot), u\right)-\sigma^{2}\left\langle\partial^{2} G(\omega, u)\right\rangle_{Q}\right) \\
& +\frac{1}{\sqrt{N}} \int_{s}^{t} \sum_{1 \leqslant j \leqslant N} \partial G\left(z_{j}(\cdot), u\right) d z_{j}(u)
\end{aligned}
$$

which can be written as

$$
\begin{aligned}
\left(G(\omega, t), \xi^{N}\right)-\left(G(\omega, s), \xi^{N}\right)= & \frac{\sigma^{2}}{2 \sqrt{N}} \int_{s}^{t} \sum_{1 \leqslant j \leqslant N}\left(\partial^{2} G\left(z_{j}(\cdot), u\right)-\left\langle\partial^{2} G(\omega, u)\right\rangle_{Q}\right) \\
& +\frac{1}{\sqrt{N}} \int_{s}^{t} \sum_{1 \leqslant j \leqslant N} \partial^{2} G\left(z_{j}(\cdot), u\right) d z_{j}(u)+\int_{s}^{t} e_{N}(G, u) d u
\end{aligned}
$$

with error term less than (4.9). We compute the square of the expectation of the difference. The right-hand side terms behave as follows

$$
\begin{gathered}
\limsup _{N \rightarrow \infty} E\left|\frac{1}{\sqrt{N}} \int_{s}^{t} \sum_{1 \leqslant j \leqslant N}\left(\partial^{2} G\left(z_{j}(\cdot), u\right)-\left\langle\partial^{2} G(\omega, u)\right\rangle_{Q}\right) d u\right|^{2} \\
\leqslant \int_{S}^{t} \limsup _{N \rightarrow \infty} E\left|\left(\partial^{2} G(\omega, u), \xi^{N}\right)\right|^{2} d u \leqslant(t-s) C\left(\partial^{2} G\right)
\end{gathered}
$$

according to Proposition 5 (we recall that $C\left(\partial^{2} G\right)$ did not depend on any particular time $t$ ). The martingale term will satisfy a similar inequality due to Lemma A.1, Eq. (A.2). We use the fact that

$$
\begin{aligned}
& \left(\frac{1}{\sqrt{N}} \int_{s}^{t} \sum_{1 \leqslant j \leqslant N} \partial G\left(z_{j}(\cdot), u\right) d z_{j}(u)\right)^{2}-\frac{1}{N} \int_{s}^{t} \sum_{1 \leqslant j \leqslant N}\left(\partial G\left(z_{j}(\cdot), u\right)\right)^{2} \sigma_{N, i}^{2}(u) d u \\
& \quad-\frac{1}{N^{2}} \int_{s}^{t} \sum_{1 \leqslant i \neq j \leqslant N}\left(\partial G\left(z_{i}(\cdot), u\right)\right)\left(\partial G\left(z_{j}(\cdot), u\right)\right)\left(N k_{i, j}^{N}\right)(u) d u
\end{aligned}
$$

is a martingale. We can substitute $\sigma_{N, i}^{2}(u)$ with $\sigma^{2}$ and $N k_{i, j}^{N}(u)$ by $\gamma \sigma^{2}$ due to (4.9) and (4.10). In the limit as $N \rightarrow \infty$, the law of large numbers (Proposition 5) yields a quadratic variation equal to exactly $\sigma_{\xi}^{2}(\partial F, \partial G)$. 
We have shown, based on Proposition 5, that for any $F, G \in \mathcal{C}(\Omega)$ we can consider a weak limit of $\left(F, \xi^{N}\right)$ and $\left(G, \xi^{N}\right)$ denoted by $Z(F)$ and $Z(G)$, respectively. Formulas (4.15) and (4.16) imply that $Z(G(\omega, t))$ satisfies the inductive SPDE:

$$
Z(G(\omega, t))-Z\left(G\left(\omega, t^{\prime}\right)\right)=\frac{\sigma^{2}}{2} \int_{t^{\prime}}^{t} Z\left(\partial^{2} G(\omega, s)\right) d s+\sigma \int_{t^{\prime}}^{t} \sqrt{\check{q}(\partial G(s), \partial G(s))} d \beta(s)
$$

with $q(F, G)$ defined in (4.2). The calculations involved in determining the covariance are direct consequences of Proposition 4. The analogous formula holds for $F$ and the product $\bar{u}(F, G)=Z(F(\omega, t)) Z(G(\omega, t))$ satisfies the inductive property that

$$
\begin{aligned}
& \bar{u}(F(\omega, t), G(\omega, t))-\bar{u}\left(F\left(\omega, t^{\prime}\right), G\left(\omega, t^{\prime}\right)\right) \\
& \quad-\frac{\sigma^{2}}{2} \int_{t^{\prime}}^{t}\left(\bar{u}\left(\partial^{2} F(\omega, s), G(\omega, s)\right)+\bar{u}\left(F(\omega, s), \partial^{2} G(\omega, s)\right)+2 \breve{q}(\partial F(\omega, s), \partial G(\omega, s))\right) d s
\end{aligned}
$$

is an $\left(P, \mathcal{F}_{t}\right)$-martingale. Taking the expected value, Eq. (4.18) becomes (3.2).

Proof of Theorem 2. Proposition 5 proves that the fluctuation random fields $\left\{\xi^{N}\right\}_{N>0}$ are tight in the weak* topology over the space of special functions $(\mathcal{E} \cup \mathcal{S})(\Omega)$. Proposition 6 proves the conclusion of the theorem for functions in the special class $(\mathcal{E} \cup \mathcal{S})(\Omega)$ based on Theorem 4 in Section 3 after identifying the covariance function from Proposition 4.

\section{Extension to $C_{b}^{1}(\Omega)$}

This section proves Theorem 3 via Proposition 10. Suppose we can figure out the covariance of the limiting random field in the central limit theorem for cylinder functions. If the covariance is continuous in some norm on $C_{b}^{1}(\Omega)$, then we can define directly the limit as a Gaussian on the completion of the new space. Identifying the limit in Theorem 2 does not provide us with a class of functions for which the central limit theorem takes place, except for Schwartz or exponential class cylinder functions, which may be a rather poor space. However, Proposition 9, together with the density result from the next theorem overcome this difficulty and enable us to prove Theorem 3 in an infinite dimensional setting using the test function space $C_{b}^{1}(\Omega)$.

The proof of Proposition 9 requires that we evaluate the $L^{2}$ norm of the renormalized differences (amplified by a factor of $\sqrt{N}$ ) between the martingales $\left\{z_{i}(\cdot)\right\}_{1 \leqslant i \leqslant N}$ and the limiting Brownian motions distributed according to $Q$ from the decomposition Lemma 2, which is the missing link between Theorem 5 and Proposition 9 - see also the comment related to (2.15). It seems hard to connect the supremum norm of the path space $\Omega$ and the expectation of its square except by assuming that the error terms are essentially martingales with quadratic variation of $\mathrm{O}(1 / N)$ and using Doob's inequality with an exponential bound guaranteed by Proposition 8. Proposition 10, which finishes the proof of Theorem 3 is based on Proposition 9.

Theorem 5. Let $G \in C_{b}^{1}(\Omega)$. If $K$ is a compact with $K \subseteq \Omega$, then for any $\varepsilon>0$ there exists a function $G_{\varepsilon}^{S} \in \mathcal{S}(\Omega)$ such that $\left\|G_{\varepsilon}^{S}\right\|_{C_{b}^{1}} \leqslant 2\|G\|_{C_{b}^{1}}$ and

$$
\sup _{\omega \in K}\left\{\left(\left|G(\omega)-G_{\varepsilon}^{S}(\omega)\right|+\left\|\nabla \cdot G(\omega)-\nabla \cdot G_{\varepsilon}^{S}(\omega)\right\|\right)\right\}<\varepsilon
$$

The proof of Theorem 5 is in Appendix A.

Proposition 7. The space $\mathcal{S}(\Omega)$ is dense in $\mathcal{H}(\Omega, Q)$. 
Proof. $Q$ is a Brownian motion hence it is supported on the countable union of compacts of the form $K_{\alpha}=\Omega_{0} \cap$ $B(0, \alpha)$, where $B(0, \alpha)$ is the ball of radius $\alpha$ centered at $\omega=0$ and $\alpha>0$ converges to $\infty$ meanwhile $\Omega_{0}$ is the set of Hölder continuous paths $\omega \in \Omega$ with exponent $\tilde{v} \in\left(0, \frac{1}{2}\right)$. These sets are equicontinuous and bounded in the supremum norm, which implies they are compact by Arzelà's theorem. The functions $G \in C_{b}^{1}(\Omega)$ have finite $\|\cdot\|_{\mathcal{H}}$ norm. For every $G$ the measures $\left(|G(\omega)|^{2}+\|\nabla \cdot G(\omega)\|^{2}\right) d Q(\omega)$ are absolutely continuous with respect to $Q$ and

$$
\int_{\Omega_{0}}|G(\omega)|^{2}+\|\nabla \cdot G(\omega)\|^{2} d Q(\omega)=\int_{\Omega}|G(\omega)|^{2}+\|\nabla \cdot G(\omega)\|^{2} d Q(\omega),
$$

hence

$$
\lim _{\alpha \rightarrow \infty} \int_{K_{\alpha}^{c}}|G(\omega)|^{2}+\|\nabla \cdot G(\omega)\|^{2} d Q(\omega)=0
$$

by dominated convergence. This implies that there is a compact $K_{\alpha_{0}}$ with the property

$$
\int_{K_{\alpha_{0}}^{c}} d Q(\omega)<\varepsilon \quad \text { and } \int_{K_{\alpha_{0}}^{c}}|G(\omega)|^{2}+\|\nabla \cdot G(\omega)\|^{2} d Q(\omega)<\varepsilon .
$$

Finally, for this compact we pick a $G_{\varepsilon}^{S} \in \mathcal{S}(\Omega)$ such that (5.1) and $\left\|G_{\varepsilon}^{S}\right\|_{C_{b}^{1}} \leqslant 2\|G\|_{C_{b}^{1}}$ are satisfied. This and the Schwartz inequality imply that

$$
\begin{aligned}
\left\|G-G_{\varepsilon}^{S}\right\|_{\mathcal{H}}^{2} \leqslant & \int_{K_{\alpha_{0}}}\left[\left|G(\omega)-G_{\varepsilon}^{S}(\omega)\right|^{2}+\left\|\nabla \cdot\left(G(\omega)-G_{\varepsilon}^{S}(\omega)\right)\right\|^{2}\right] d Q(\omega) \\
& +2 \varepsilon+2\left\|G_{\varepsilon}^{S}\right\|_{C_{b}^{1}}^{2} \int_{K_{\alpha_{0}}^{c}} d Q(\omega) \leqslant\left(3+8\|G\|_{C_{b}^{1}}^{2}\right) \varepsilon
\end{aligned}
$$

The claim is proven.

Lemma 1 is an independent result valid in any inner product space. It is not essential that the space be complete (hence Hilbert). We shall use the standard notation $\|\cdot\|$ for the norm. This should not be confused with the supremum norm on $\Omega$ the path space of continuous functions. The heuristic argument relating it to our proof is that the inner product is analogous to the quadratic variation.

Lemma 1. Let $\tilde{X}$ be an inner product space with inner product denoted by $(\cdot, \cdot)$. The associated norm will be denoted by $\|\cdot\|$. For any positive integer $N \in \mathbb{Z}_{+}$we shall consider a family of vectors $\left\{v_{i}^{N}\right\}_{1 \leqslant i \leqslant N}$ with the property that there exist two numbers $\sigma>0$ and $\gamma>-1$, independent of $N$ such that

$$
\lim _{N \rightarrow \infty} \sqrt{N} \max _{1 \leqslant i \leqslant N}\left|\left(v_{i}^{N}, v_{i}^{N}\right)-\sigma^{2}\right|=0
$$

and

$$
\lim _{N \rightarrow \infty} \max _{1 \leqslant i, j \leqslant N}\left|N\left(v_{i}^{N}, v_{j}^{N}\right)-\gamma \sigma^{2}\right|=0
$$

Then, for $N$ sufficiently large, the matrix $V^{N}$ with elements $V_{i j}^{N}=\left(v_{i}^{N}, v_{j}^{N}\right)$ is positive definite and there exists an orthonormal system of $N$ vectors $\left\{w_{i}^{N}\right\}_{1 \leqslant i \leqslant N}$ and a constant $C$, independent of $N$, such that

$$
\max _{1 \leqslant i \leqslant N}\left\|v_{i}^{N}-\sigma w_{i}^{N}\right\|^{2} \leqslant \frac{C}{N}
$$

Proof. The proof of Lemma 1 is in Appendix A. 
Lemma 2. Assume that conditions (2.13) and (2.14) are met. Then, for any $N \in \mathbb{Z}_{+}$there exist $N\left(P, \mathcal{F}_{t}\right)$ martingales $\left\{y_{i}^{N}(\cdot)\right\}_{1 \leqslant i \leqslant N}$ such that $\left\{\sigma^{-1} y_{i}^{N}(\cdot)\right\}_{1 \leqslant i \leqslant N}$ is a standard $N$-dimensional Brownian motion with the property that the renormalized differences $d_{i}^{N}(\cdot)=\sqrt{N}\left(z_{i}^{N}(\cdot)-y_{i}^{N}(\cdot)\right)$ are continuous square-integrable martingales with quadratic variations $\left\langle d_{i}^{N}, d_{i}^{N}\right\rangle(\cdot)$ for which there exists a constant $C$, independent of $N$, the time $t \in[0, T]$ and the indices $1 \leqslant i \leqslant N$, such that

$$
0 \leqslant \frac{d}{d t}\left\langle d_{i}^{N}, d_{i}^{N}\right\rangle(t) \leqslant C
$$

$P$-almost surely.

Remark. The martingale representation theorem implies that the quadratic variation process of a square-integrable martingale relative to the filtration $\left\{\mathcal{F}_{t}\right\}_{t} \geqslant 0$ is absolutely continuous $P$-almost surely with respect to the Lebesgue measure.

Proof of Lemma 2. The martingale representation theorem (in [8], p. 84) and the fact that the covariance matrix of the martingales $\left\{z_{i}^{N}(\cdot)\right\}_{1 \leqslant i \leqslant N}$ is positive definite (Lemma 1) ensure the existence of a system of $N$ independent Brownian motions $\beta_{1}(\cdot), \ldots, \beta_{N}(\cdot)$ on $(\mathfrak{W}, P, \mathcal{F})$, adapted to the original filtration $\left\{\mathcal{F}_{t}\right\}_{t} \geqslant 0$, and a set of progressively measurable square integrable system of functions $\psi_{i l}^{N}(t)$, with $1 \leqslant l \leqslant N$ and $1 \leqslant i \leqslant N$ such that, $P$-almost surely,

$$
z_{i}^{N}(t)=z_{i}^{N}(0)+\sum_{l=1}^{N} \int_{0}^{t} \psi_{i l}^{N}(s) d \beta_{l}(s) .
$$

Let $H_{N}$ be the subspace generated by the martingales $\left\{\beta_{i}(\cdot)\right\}_{1 \leqslant i \leqslant N}$ in $L^{2}(\Omega, P)$, that is, the completion under the $L^{2}(\mathfrak{W}, P)$ norm of the martingales of the form

$$
M(t)=M(0)+\sum_{l=1}^{N} \int_{0}^{t} r_{l}(s) d \beta_{l}(s)
$$

with $r_{l}(s), 1 \leqslant l \leqslant N$, a family of bounded progressively measurable functions with respect to $\left\{\mathcal{F}_{t}\right\}_{t} \geqslant 0$. Let $M(\cdot)$ be an element from $H_{N} . M(t)$ can be written in terms of square integrable functions $r_{l}(s)$ as in (5.5). For every $u \in[0, T]$ we shall define the mapping $S_{u}$ from $H_{N}$ to $\mathbb{R}^{N}$ by

$$
S_{u}(M(\cdot))=\left(r_{1}(u), \ldots, r_{N}(u)\right) .
$$

If $M_{1}(\cdot)$ and $M_{2}(\cdot)$ are two elements of $H_{N}$, the quadratic variation will be the time integral of the Euclidian inner product on $\mathbb{R}^{N}$ for the vectors $S_{u}\left(M_{1}(\cdot)\right)$ and $S_{u}\left(M_{2}(\cdot)\right)$. The martingale representation theorem implies that any square-integrable martingale will have an absolutely continuous quadratic variation $P$-almost surely. As a consequence, we can evaluate the derivative of the quadratic variation process for any martingale in $H_{N}$ directly from the Euclidian norm of the vector $S_{u}(M(\cdot))$. Let $N^{-1 / 2} d_{i}^{N}(\cdot)$, for $1 \leqslant i \leqslant N$ be the differences between the original martingales and the orthonormal set of martingales produced by Lemma 1 . All the transformations involved in the procedure are progressively measurable. We always can start our orthonormal set of martingales and the original set of martingales from the same points so that the differences actually start at zero. Assumptions (2.13) and (2.14) imply that the conditions of Lemma 1 are satisfied with nonrandom bounds independent from $N$ and the indices $1 \leqslant i \leqslant N$ for all $\left\{S_{u}\left(z_{i}^{N}(\cdot)\right)\right\}$. This implies that the difference processes $\left\{d_{i}^{N}(\cdot)\right\}_{1 \leqslant i \leqslant N}$ will satisfy the property

$$
0 \leqslant \frac{d}{d u}\left\langle d_{i}^{N}, d_{i}^{N}\right\rangle(u) \leqslant C
$$

$P$-almost surely for some universal constant $C$. This proves the lemma. 
Proposition 8. Let $\left\{m^{N}(\cdot)\right\}_{N}$ be a family of continuous square integrable martingales with respect to $\left(P,\left\{\mathcal{F}_{t \geqslant 0}\right\}_{t} \geqslant 0\right)$ starting from a sequence of uniformly bounded values $m^{N}(0)$. For any $N \in \mathbb{Z}_{+}$there exist random functions $a_{N}(t)$ and a constant $K>0$ independent from $N$, such that the cross-variation process of $m^{N}(\cdot)$ satisfies

$$
\left\langle m^{N}, m^{N}\right\rangle(t)=\int_{0}^{t} a_{N}(s) d s
$$

with $\left|a_{N}(t)\right| \leqslant K$ P-almost surely. Then, for any $t>0$ and $\ell \in \mathbb{R}$,

$$
\limsup _{N \rightarrow \infty} E\left[\exp \left(\ell\left|m^{N}(t)\right|\right)\right]<\infty .
$$

Proof. Since $\exp (|z|) \leqslant \exp (z)+\exp (-z)$ it is enough to check what happens for $\exp \left(\ell m^{N}(t)\right)$ with $\ell \in \mathbb{R}$. We apply the Itô formula to the function $z \rightarrow \exp (\ell z)$ and obtain

$$
\exp \left(\ell m^{N}(t)\right)-\exp \left(\ell m^{N}(0)\right)-\frac{\ell^{2}}{2} \int_{0}^{t} \exp \left(\ell m^{N}(s)\right) a_{N}(s) d s
$$

is a martingale with respect to the same filtration and $P$. The expected value shows that if we denote $w_{N}(t)=$ $E\left[\exp \left(\ell m^{N}(t)\right)\right]$ then

$$
w_{N}(t) \leqslant w_{N}(0)+\frac{K \ell^{2}}{2} \int_{0}^{t} w_{N}(s) d s
$$

which, in its turn, proves that $w_{N}(t) \leqslant w_{N}(0) \exp \left(K \ell^{2} t / 2\right)$ for all $0 \leqslant t \leqslant T$. Since $w_{N}(0)$ and $t$ are bounded quantities we are done.

Remark. Proposition 8 is much stronger than what we need, that is the $r$-integrability of $m^{N}(t)$ for some $r \geqslant 4$.

Proposition 9. There exists a constant $C$ depending only on $T$ such that for any $G \in C_{b}^{1}(\Omega)$

$$
\limsup _{N \rightarrow \infty} E\left[\left|\left(G, \xi^{N}\right)\right|^{2}\right] \leqslant C\|G\|_{\mathcal{H}}^{2} .
$$

Proof. Let $y_{i}^{N}(\cdot), 1 \leqslant i \leqslant N$, be the independent Brownian motions from Lemma 2 .

$$
\frac{1}{\sqrt{N}} \sum_{1}^{N}\left(G\left(z_{i}^{N}(\cdot)\right)-\langle G\rangle_{Q}\right)=\frac{1}{\sqrt{N}} \sum_{1}^{N}\left(G\left(z_{i}^{N}(\cdot)\right)-G\left(y_{i}^{N}(\cdot)\right)\right)+\frac{1}{\sqrt{N}} \sum_{1}^{N}\left(G\left(y_{i}^{N}(\cdot)\right)-\langle G\rangle_{Q}\right) .
$$

Let's denote $d_{i}^{N}(\cdot)=\sqrt{N}\left(z_{i}^{N}(\cdot)-y_{i}^{N}(\cdot)\right)$. We decompose the first term into

$$
(I)=\frac{1}{\sqrt{N}}\left(\sum_{1}^{N} G\left(z_{i}^{N}(\cdot)\right)-G\left(y_{i}^{N}(\cdot)\right)-\frac{1}{\sqrt{N}} \nabla G_{d_{i}^{N}(\cdot)}\left(y_{i}^{N}(\cdot)\right)\right)
$$

and

$$
(I I)=\frac{1}{N} \sum_{1}^{N} \nabla_{d_{i}^{N}(\cdot)} G\left(y_{i}^{N}(\cdot)\right) .
$$


Again, we look at the two terms in this formula separately. We recall (2.1) from Definition 1 and see that we can write an upper bound for $|(I)|$ as

$$
\frac{1}{\sqrt{N}} \sum_{1}^{N}\left|G\left(z_{i}^{N}(\cdot)\right)-G\left(y_{i}^{N}(\cdot)\right)-\frac{1}{\sqrt{N}} \nabla G_{d_{i}^{N}(\cdot)}\left(y_{i}^{N}(\cdot)\right)\right| \leqslant \sqrt{N} c\left(G, \frac{1}{\sqrt{N}}\right)\left(\frac{1}{N} \sum_{1}^{N}\left\|d_{i}^{N}(\cdot)\right\|^{2}\right) .
$$

This quantity goes to zero as $N \rightarrow \infty$ as soon as the martingales $d_{i}^{N}(\cdot)$ have a finite second moment. This is guaranteed by Proposition 8 (the uniformity in $N$ and $j$ ) and Doob's maximal inequality regarding any $L^{r}$ norm of martingales $(r>1)$ for all $1 \leqslant i \leqslant N$ :

$$
\left(E\left[\left|\sup _{0 \leqslant t \leqslant T} d_{i}(t)\right|^{r}\right]\right)^{1 / r} \leqslant \frac{r}{r-1}\left(E\left[\left|d_{i}(T)\right|^{r}\right]\right)^{1 / r} .
$$

The term

$$
E\left[|(I I)|^{2}\right]=E\left[\frac{1}{N} \sum_{1}^{N} \frac{\nabla_{\sqrt{N}\left(z_{i}^{N}(\cdot)-y_{i}^{N}(\cdot)\right)} G\left(y_{i}^{N}(\cdot)\right)}{\left\|\sqrt{N}\left(z_{i}^{N}(\cdot)-y_{i}^{N}(\cdot)\right)\right\|} \cdot\left\|\sqrt{N}\left(z_{i}^{N}(\cdot)-y_{i}^{N}(\cdot)\right)\right\|\right]^{2}
$$

can be estimated if we expand the square after taking the supremum norm for the linear operators $\nabla \cdot G\left(y_{i}^{N}(\cdot)\right)($ as in Definition 1)

$$
\begin{gathered}
E\left[\frac{1}{N} \sum_{1}^{N}\left\|\nabla \cdot G\left(y_{i}(\cdot)\right)\right\|\left\|d_{i}^{N}(\cdot)\right\|\right]^{2} \leqslant E\left[\frac{1}{N^{2}} \sum_{1}^{N}\left(\left\|\nabla \cdot G\left(y_{i}(\cdot)\right)\right\|\left\|d_{i}^{N}(\cdot)\right\|\right)^{2}\right] \\
+\frac{1}{N^{2}} \sum_{1 \leqslant i \neq j \leqslant N} E\left[\left(\left\|\nabla \cdot G\left(y_{i}(\cdot)\right)\right\|\left\|\nabla \cdot G\left(y_{j}(\cdot)\right)\right\|\right)\left(\left\|d_{i}^{N}(\cdot)\right\|\left\|d_{j}^{N}(\cdot)\right\|\right)\right] .
\end{gathered}
$$

The first term can be bounded by using the supremum $c_{1}(G)$ of all $\|\nabla \cdot G(\omega(\cdot))\|$ (Definition 1$)$. The bound is

$$
c_{1}(G)^{2} \frac{1}{N^{2}} \sum_{1 \leqslant i \neq j \leqslant N} E\left[\left(\left\|d_{i}^{N}(\cdot)\right\|^{2}\right)\right]=\mathrm{O}\left(\frac{1}{N}\right)
$$

The second term can be bounded in terms of the $L^{2}$ norm of $\left\|\nabla \cdot G\left(y_{i}(\cdot)\right)\right\|$ due to the crucial fact that $y_{i}(\cdot)$ and $y_{j}(\cdot)$ are independent Brownian motions by construction. The upper bound is obtained by Schwarz's inequality

$$
\begin{aligned}
& \frac{1}{N^{2}} \sum_{1 \leqslant i \neq j \leqslant N}\left(E\left[\left(\left\|\nabla \cdot G\left(y_{i}(\cdot)\right)\right\|^{2}\left\|\nabla \cdot G\left(y_{j}(\cdot)\right)\right\|^{2}\right)\right]\right)^{1 / 2}\left(E\left[\left(\left\|d_{i}^{N}(\cdot)\right\|\left\|d_{j}^{N}(\cdot)\right\|\right)^{2}\right]\right)^{1 / 2} \\
& \quad \leqslant\|G\|_{\mathcal{H}}^{2} \frac{1}{N^{2}} \sum_{1 \leqslant i \neq j \leqslant N} D_{i} D_{j},
\end{aligned}
$$

where $D_{i}=\left(E\left[\left\|d_{i}(\cdot)\right\|^{4}\right]\right)^{1 / 4}$ for all $1 \leqslant i \leqslant N$. These norms are bounded. We use again Doob's inequality (5.8) and Proposition 8 to ensure uniformity in both $N$ and $i$. Finally

$$
\limsup _{N \rightarrow \infty} E\left[\frac{1}{\sqrt{N}} \sum_{1}^{N}\left(G\left(y_{i}^{N}(\cdot)-\langle G\rangle_{Q}\right)\right)\right]^{2} \leqslant E\left[G^{2}-(E[G])^{2}\right] \leqslant 2\|G\|_{\mathcal{H}}^{2}
$$

due to the central limit theorem variance for independent random variables. The last two bounds provide an upper bound of the form $C \cdot\|G\|_{\mathcal{H}}^{2}$ with the constant independent from $N$ and $G$. This concludes the proof of the proposition. 
Proposition 10. Let $\left\{\xi^{N}\right\}_{N>0}$ be a family of random fields on $C_{b}^{1}(\Omega)$ defined in (2.11) and $q(\cdot, \cdot) \in B(\mathcal{H}(\Omega, Q))$ be the bilinear form (2.12). Then, for any pair $F^{S}, G^{S} \in \mathcal{S}(\Omega)$

(i) $\left(F^{S}, \xi^{N}\right)$ and $\left(G^{S}, \xi^{N}\right)$ converge weakly to mean zero normal random variables with covariance $q\left(F^{S}-\right.$ $\left.\left\langle F^{S}\right\rangle_{Q}, G^{S}-\left\langle G^{S}\right\rangle_{Q}\right)$

(ii) $\lim _{N \rightarrow \infty} E\left[\left(F^{S}, \xi^{N}\right)\left(G^{S}, \xi^{N}\right)\right]=q\left(F^{S}-\left\langle F^{S}\right\rangle_{Q}, G^{S}-\left\langle G^{S}\right\rangle_{Q}\right)$.

(iii) For any $G \in C_{b}^{1}(\Omega)$, the sequence $\left\{\left(G, \xi^{N}\right)\right\}_{N>0}$ is tight, and if $F, G \in C_{b}^{1}(\Omega)$ then the random variables $\left(F, \xi^{N}\right),\left(G, \xi^{N}\right)$ converge weakly to centered Gaussians with covariance $q\left(F-\langle F\rangle_{Q}, G-\langle G\rangle_{Q}\right)$. As a consequence, the unique limit $\xi$ of the sequence $\left\{\xi^{N}\right\}_{N>0}$ is a centered Gaussian random field on $\mathcal{H}(\Omega, Q)$ with the same covariance.

Proof. Proposition 6 and Theorem 4 show that the sequence $\left\{\left(G^{S}, \xi^{N}\right)\right\}_{N>0}$ converges weakly to a centered normal random variable $Z\left(G^{S}\right)$. Moreover, $Z\left(G^{S}\right)$ has variance equal to $q\left(G^{S}-\left\langle G^{S}\right\rangle_{Q}, G^{S}-\left\langle G^{S}\right\rangle_{Q}\right)$. This proves (i) and (ii).

Let $l \in \mathbb{Z}_{+}$. Theorem 5 and Proposition 7 indicate that for any $G \in C_{b}^{1}(\Omega)$ we can choose the appropriate $G_{\varepsilon}^{S}$ for $\varepsilon=1 / l$, which we shall denote by $G_{l}^{S}$, such that

$$
\left\|G-G_{l}^{S}\right\|_{\mathcal{H}}^{2} \leqslant \frac{1}{l}
$$

Eq. (5.10) implies that $\lim _{l \rightarrow \infty}\left\|G_{l}^{S}-G\right\|_{\mathcal{H}}=0$, which also guarantees that $G_{l}^{S}$ are uniformly square integrable, hence the sequence of centered Gaussian random variables $\left\{Z\left(G_{l}^{S}\right)\right\}_{l \in \mathbb{Z}_{+}}$is tight. Let $Z^{S}(G)$ be a limit point. This has to be a centered Gaussian (we can look at the characteristic function of the Gaussians) and its variance will be (see [15])

$$
\sigma^{2}\left(Z^{S}(G)\right)=\lim _{l \rightarrow \infty} q\left(G_{l}^{S}-\left\langle G_{l}^{S}\right\rangle_{Q}, G_{l}^{S}-\left\langle G_{l}^{S}\right\rangle_{Q}\right)=q\left(G-\langle G\rangle_{Q}, G-\langle G\rangle_{Q}\right) .
$$

We already know from (5.7) that $\left\{\left(G, \xi^{N}\right)\right\}_{N>0}$ is tight as well. Let $Z(G)$ be a limit point of $\left\{\left(G, \xi^{N}\right)\right\}_{N>0}$. We can restrict ourselves without loss of generality to subsequences of $\{l\}$ and $\{N\}$ such that $Z\left(G_{l}^{S}\right) \Rightarrow Z^{S}(G)$ and $\left(G, \xi^{N}\right) \Rightarrow Z(G)$.

For $\alpha \in \mathbb{R}$ we consider

$$
\begin{aligned}
& \left|E\left[\mathrm{e}^{-\mathrm{i} \alpha Z^{S}(G)}\right]-E\left[\mathrm{e}^{-\mathrm{i} \alpha Z(G)}\right]\right| \\
& \quad \leqslant\left|E\left[\mathrm{e}^{-\mathrm{i} \alpha Z^{S}(G)}\right]-E\left[\mathrm{e}^{-\mathrm{i} \alpha Z\left(G_{l}^{S}\right)}\right]\right|+\left|E\left[\mathrm{e}^{-\mathrm{i} \alpha Z\left(G_{l}^{S}\right)}\right]-E\left[\mathrm{e}^{-\mathrm{i} \alpha\left(G_{l}^{S}, \xi^{N}\right)}\right]\right| \\
& \quad+\left|E\left[\mathrm{e}^{-\mathrm{i} \alpha\left(G_{l}^{S}, \xi^{N}\right)}\right]-E\left[\mathrm{e}^{-\mathrm{i} \alpha\left(G, \xi^{N}\right)}\right]\right|+\left|E\left[\mathrm{e}^{-\mathrm{i} \alpha\left(G, \xi^{N}\right)}\right]-E\left[\mathrm{e}^{-\mathrm{i} \alpha Z(G)}\right]\right| .
\end{aligned}
$$

The third term in (5.13) has the upper bounds

$$
\begin{aligned}
& \left|E\left[\mathrm{e}^{-\mathrm{i} \alpha\left(G_{l}^{S}, \xi^{N}\right)}\right]-E\left[\mathrm{e}^{-\mathrm{i} \alpha\left(G, \xi^{N}\right)}\right]\right| \\
& \quad \leqslant E\left[\left|\mathrm{e}^{-i \alpha\left(G-G_{l}^{S}, \xi^{N}\right)}-1\right|\right] \\
& \quad \leqslant 2 E\left[\sin \left(\frac{\left(G-G_{l}^{S}, \xi^{N}\right)}{2}\right)\right] \leqslant E\left[\left|\left(G-G_{l}^{S}, \xi^{N}\right)\right|\right] \leqslant E\left[\left|\left(G-G_{l}^{S}, \xi^{N}\right)\right|^{2}\right]^{1 / 2} .
\end{aligned}
$$

If we let $N \rightarrow \infty$ we obtain that (5.12) is less than

$$
\left|E\left[\mathrm{e}^{-\mathrm{i} \alpha Z^{S}(G)}\right]-E\left[\mathrm{e}^{-\mathrm{i} \alpha Z\left(G_{l}^{S}\right)}\right]\right|+\sqrt{C}\left\|G-G_{l}^{S}\right\|_{\mathcal{H}},
$$

where $C$ is the constant in (5.7). We obtain that $E\left[\mathrm{e}^{-\mathrm{i} \alpha Z^{S}(G)}\right]=E\left[\mathrm{e}^{-\mathrm{i} \alpha Z(G)}\right]$ after $l \rightarrow \infty$. The left hand term is the Fourier transform of a centered Gaussian, which proves that the right hand side is a centered Gaussian as well for any limit point of $\left(G, \xi^{N}\right)_{N>0}$ and any $G \in C_{b}^{1}(\Omega)$. On the other hand, we have shown that $Z(G)$ has the same 
distribution as $Z^{S}(G)$, a weak limit of Gaussians $Z\left(G_{l}^{S}\right)$ as $l \rightarrow \infty$, which implies that the variance of the limit is the limit of the variances (5.11). We can repeat this reasoning for $G=c_{1} G_{1}+c_{2} G_{2}$ with arbitrary constants $c_{1}, c_{2}$ and $G_{1}, G_{2} \in C_{b}^{1}(\Omega)$ and conclude the proof by polarization.

Proof of Theorem 3. We only have to apply Proposition 10 to the random fields $\left\{\xi^{N}\right\}_{N>0}$ from Theorem 2 from Section 4.

\section{Acknowledgements}

The author would like to thank Professor S.R.S. Varadhan for suggesting the original (harder) problem and also for simplifying the proof of Lemma 1 .

\section{Appendix A}

Proof of Theorem 1. In this case simple product functions are sufficient to prove the theorem. We may choose them in $\mathcal{S}(\Omega)$ without any loss of generality. We shall proceed by induction on the number $m$ of time marginals present in $G$. For $m=1$ the martingale part vanishes as $N \rightarrow \infty$ in Itô formula. The integrand of the $d t$ term is uniformly bounded by the supremum norm of $\partial^{2} G$. The error terms have bounds of order inferior to $N^{-1}$, as prescribed by (4.9). This implies the tightness. Any limit point will be deterministic, since the martingale part vanishes as $N \rightarrow \infty$ and we can easily check that it must verify the same weak PDE (the heat equation) as $E[G(\omega, t)]$. The details of the proof of this type of result can be found, for example, in [11] in Chapter 4, and also in [5]. The same reasoning applies when we perform the induction step over $m$, since we are allowed to start over from an arbitrary initial profile at time $t^{\prime}=t_{m-1}$. We can pass to functions of $C_{b}(\Omega)$ class due to the fact that

$$
\limsup _{N \rightarrow \infty} E\left[\left|\frac{1}{N} \sum_{i=1}^{N}\left(G\left(z_{i}^{N}(\cdot)\right)-\langle G\rangle_{Q}\right)\right|^{2}\right] \leqslant 2\left(\sup _{\omega \in \Omega}|G(\omega)|\right)^{2} .
$$

This concludes the proof.

Lemma A.1. For any $G \in \mathcal{C}(\Omega)$ as in (2.4)

$$
\left.\left.\lim _{N \rightarrow \infty} E\left[\sup _{t^{\prime} \leqslant t \leqslant T} \mid \frac{1}{N} \sum_{i=1}^{N}\left(G\left(z_{i}^{N}, t\right)\right)-\langle G(\omega, t)\rangle_{Q}\right)\right|^{2}\right]=0 .
$$

Proof. Because of (A.1), it is sufficient to prove the lemma for cylinder functions $G \in \mathcal{S}_{\text {cyl }}(\Omega)$. We write $G(\omega(\cdot))=g\left(\omega\left(t_{0}\right), \ldots, \omega\left(t_{m}\right)\right)$ with $g$ in the Schwartz class on $\mathbb{R}^{m+1}$ and let $t_{m}$ be denoted by $t$ and $t_{m-1}$ by $t^{\prime}$ for simplification.

With the convention of notation from (2.4), we first establish (A.2) for a fixed time $t \in\left[t^{\prime}, T\right]$. This is a consequence of the inductive proof from Theorem 1 . Since the test function is uniformly bounded and the quadratic variation of the martingale part vanishes as $N \rightarrow \infty$ we can adapt the same argument by squaring the differences and show (A.2) for every fixed time. In order to prove uniformity, assume that there is a sequence of times such that the absolute value in (A.2) exceeds a constant $c>0$. Since the interval $[0, T]$ is compact, there is a subsequence of times converging to some $t^{\prime \prime} \in[0, T]$. However, the functionals are continuous in time at $t^{\prime \prime}$ and $G(\omega, t)$ approaches the value at $t^{\prime \prime}$ according to the estimate 


$$
\begin{aligned}
& \left.\left.\limsup _{N \rightarrow \infty} E\left[\mid \frac{1}{N} \sum_{i=1}^{N}\left(G\left(z_{i}^{N}, t\right)\right)-\langle G(\omega, t)\rangle_{Q}\right)-\frac{1}{N} \sum_{i=1}^{N}\left(G\left(z_{i}^{N}, t^{\prime \prime}\right)\right)-\left\langle G\left(\omega, t^{\prime \prime}\right)\right\rangle_{Q}\right)\left.\right|^{2}\right] \\
& \quad \leqslant 2 \limsup _{N \rightarrow \infty}\left\{\sup _{\bar{x}}|\partial G|^{2} E\left[\frac{1}{N} \sum_{i=1}^{N}\left|z_{i}^{N}(t)-z_{i}^{N}\left(t^{\prime \prime}\right)\right|^{2}\right]+2 T \sigma^{2} c_{1}(G)\left|t-t^{\prime \prime}\right|\right\} \\
& \leqslant c_{2}(G, T) \sigma^{2}\left|t-t^{\prime \prime}\right| .
\end{aligned}
$$

The error is independent of $N$ and of the order of magnitude of $t-t^{\prime \prime}$. The error obtained is arbitrarily small, a contradiction with the fact that the absolute value in (A.2) exceeds a constant $c>0$. This concludes the proof.

Proof of Theorem 5. Let $m \in \mathbb{Z}_{+}$. The mapping $T_{m}: \Omega \rightarrow \Omega$ is defined for each $\omega$ as the new continuous path obtained by linear interpolation between the values $\omega\left(t_{i}\right)$, for all $0 \leqslant i \leqslant m$, at the points $t_{i}=i T / m$. It follows that $T_{m}$ is linear and continuous with $\left\|T_{m}\right\| \leqslant 1$ in the supremum norm. Since $T_{m}$ is linear and continuous it is differentiable and $\nabla_{\eta} T_{m} \omega=T_{m} \eta$. We shall define the finite-dimensional norm on $\omega \in \Omega$ as

$$
\|\omega\|_{m}=\max _{0 \leqslant i \leqslant m}\left|\omega\left(t_{i}\right)\right| .
$$

Any compact set in $\Omega$ is uniformly bounded and equicontinuous by Arzelà-Ascoli theorem as in [3]. We can assume that $|\omega(t)| \leqslant M$ for all $t \in[0, T]$ and all $\omega \in K$. The fact that $G \in C_{b}^{1}(\Omega)$ (Definition 1 ) and $K$ is a compact in $\Omega$ implies that for any $\varepsilon>0$ there exists a $\delta=\delta(\varepsilon)$ such that

$$
\left|G(\omega)-G\left(\omega^{\prime}\right)\right|+\left\|\nabla \cdot G(\omega)-\nabla \cdot G\left(\omega^{\prime}\right)\right\|<\varepsilon
$$

if $\omega^{\prime}(\cdot)$ and $\omega(\cdot)$ are in $K$ and $\sup _{0 \leqslant t \leqslant T}\left|\omega(t)-\omega^{\prime}(t)\right|<3 \delta$. We are free to choose $\delta<\min \left(\varepsilon / 2, \varepsilon /\|G\|_{C_{b}^{1}}\right)$. For any $\delta>0$ there exists an $m \in \mathbb{Z}_{+}$such that

$$
|t-s|<\frac{1}{m} \Rightarrow|\omega(t)-\omega(s)|<\delta
$$

uniformly in $\omega \in K$.

For $\varepsilon>0$ we shall choose a covering $K \subseteq \bigcup_{\omega \in K} B(\omega, \delta)$ of the compact $K$ with balls of radius $\delta=\delta(\varepsilon)$ in the uniform norm topology of $\Omega$ and extract a finite subcovering with centers at $\omega_{j}(\cdot), j \in J_{\varepsilon}$, where $J_{\varepsilon}$ is a finite set depending only on $K$ and $\varepsilon$. For every $\bar{x}=\left(x_{0}, x_{1}, \ldots, x_{m}\right) \in \mathbb{R}^{m+1}$ we determine the path $\omega_{\bar{x}}$ as the linear interpolation between the values (for $0 \leqslant i \leqslant m$ ), that is $\omega_{\bar{x}}\left(t_{i}\right)=x_{i}$. Let $K_{\delta, j}^{x}=\left\{\bar{x}:\left\|\omega_{\bar{x}}-\omega_{j}\right\|_{m} \leqslant \delta\right\}$ be the $\mathbb{R}^{m+1}$-cube of size $2 \delta$ and $K_{\delta}^{x}=\bigcup_{j \in J_{\varepsilon}} K_{\delta, j}^{x}$. We shall construct a function $g_{\varepsilon}(\bar{x})$ on $\mathbb{R}^{m+1}$ by piecing together the following mappings. For $m$ as in (A.4) and $j \in J_{\varepsilon}$, let

$$
g_{m, j}(\bar{x})=G\left(\omega_{j}\right)+\nabla_{T_{m}\left(\omega_{\bar{x}}-\omega_{j}\right)} G\left(\omega_{j}\right)
$$

on each $\mathbb{R}^{m+1}$-cube $K_{\delta, j}^{x}$ and zero everywhere else. Then, we define

$$
g_{\varepsilon}(\bar{x})=g_{m, j}(\bar{x}) \quad \text { if }\left\|\omega_{\bar{x}}-\omega_{j}\right\|_{m}=\min _{j^{\prime} \in J_{\varepsilon}}\left\|\omega_{\bar{x}}-\omega_{j^{\prime}}\right\|_{m}
$$

with the understanding that if a point falls on the hypersurface where two or more indices achieve the maximum we select the smaller index $j^{\prime}$. This fact will not affect the construction due to the mollification we do next. The function $g_{\varepsilon}(\bar{x})$ is piecewise smooth and uniformly bounded by

$$
\left|g_{m, j}(\bar{x})\right| \leqslant \sup _{\omega \in \Omega}|G(\omega)|+\sup _{\omega \in \Omega}\|\nabla \cdot G(\omega)\| \delta \leqslant\|G\|_{C_{b}^{1}} .
$$

The gradient of $g_{\varepsilon}(\bar{x})$ can only be one of the linear mappings on $\mathbb{R}^{m+1}$ from the finite family of bounded linear operators $\nabla_{T_{m}\left(\omega_{\bar{x}}-\omega_{j}\right)} G\left(\omega_{j}\right)$. They are naturally bounded in the supremum norm of continuous linear operators on $\mathbb{R}^{m+1}$ by $\sup _{\omega \in \Omega}\|\nabla . G(\omega)\|$. One can write that

$$
\sup _{\bar{x} \in \mathbb{R}^{m+1}}\left(\left|g_{\varepsilon}(\bar{x})\right|+\left\|\nabla g_{\varepsilon}(\bar{x})\right\|\right) \leqslant \sup _{\omega \in \Omega}|G(\omega)|+2\|\nabla \cdot G(\omega)\|
$$


wherever the gradient is defined and note that $\operatorname{supp}\left(g_{\varepsilon}(\bar{x})\right)$ is a compact included in $K_{\delta}^{x} \subseteq[-M-1, M+1]^{m+1}$ in $\mathbb{R}^{m+1}$.

For an arbitrary $\rho>0$ we can construct a regularized version of $g_{\varepsilon}(\bar{x})$ by convolution with $\phi_{m, \rho}(\bar{x})=$ $\rho^{-m-1} \phi\left(\rho^{-1}\|(\bar{x})\|_{\mathbb{R}^{m+1}}\right)$ where $\phi(x)=k_{0} \exp \left(1 /\left(x^{2}-1\right)\right)$ if $|x|<1$ and identically zero outside the unit interval and $\|\cdot\|_{\mathbb{R}^{m+1}}$ is the Euclidian norm on $\mathbb{R}^{m+1}$. The constant $k_{0}$ normalizes $\phi(x)$ so that the integral equals one. Let

$$
g_{\varepsilon}^{S}(\bar{x})=\left(g_{\varepsilon} * \phi_{m, \rho}\right)(\bar{x}) .
$$

We choose $\rho=\rho(\varepsilon)<\delta / 2$ to make sure that the function $g_{\varepsilon}(\bar{x})$ vanishes outside the compact $K_{2 \delta}^{x} \subseteq \mathbb{R}^{m+1}$. We shall use the observation that the convolution with $\phi_{m, \rho}$ is a contraction in the following sense. If $g(\bar{x})$ is a piecewise smooth function on $\mathbb{R}^{m+1}$ and $g^{S}(\bar{x})=\left(g * \phi_{m, \rho}\right)(\bar{x})$ then, for any pair of points $\bar{x}^{\prime}$ and $\bar{x}^{\prime \prime}$ from $\mathbb{R}^{m+1}$,

$$
\left|g^{S}\left(\bar{x}^{\prime}\right)-g^{S}\left(\bar{x}^{\prime \prime}\right)\right| \leqslant \sup _{\bar{y}^{\prime} \in B\left(\bar{x}^{\prime}, \rho\right), \bar{y}^{\prime \prime} \in B\left(\bar{x}^{\prime \prime}, \rho\right)}\left|g\left(\bar{y}^{\prime}\right)-g\left(\bar{y}^{\prime \prime}\right)\right|
$$

and

$$
\left\|\nabla g^{S}\left(\bar{x}^{\prime}\right)-\nabla g^{S}\left(\bar{x}^{\prime \prime}\right)\right\| \leqslant \sup _{\bar{y}^{\prime} \in B\left(\bar{x}^{\prime}, \rho\right), \bar{y}^{\prime \prime} \in B\left(\bar{x}^{\prime \prime}, \rho\right)}\left\|\nabla g\left(\bar{y}^{\prime}\right)-\nabla g\left(\bar{y}^{\prime \prime}\right)\right\| .
$$

This property equally allows us to estimate both

$$
\left|g^{S}(\bar{x})\right| \leqslant \sup _{\bar{y} \in B(\bar{x}, \rho)}|g(\bar{y})|, \quad\left\|\nabla g^{S}(\bar{x})\right\| \leqslant \sup _{\bar{y} \in B(\bar{x}, \rho)}\|\nabla g(\bar{y})\| .
$$

We define

$$
\widetilde{G}_{\varepsilon}^{S}(\omega)=g_{\varepsilon}^{S}\left(\omega\left(t_{0}\right), \ldots, \omega\left(t_{m}\right)\right) .
$$

The function $g_{\varepsilon}^{S}$ is of Schwartz class on $\mathbb{R}^{m+1}$ but not of cylinder type. Any such function can be approximated uniformly including its derivatives on any compact by a linear combination of cylinder-type functions of Schwartz class. We shall show this fact at the end of the proof. The norm $\|\omega\|_{m}$ determines a family of sets $B_{m}\left(\omega_{j}, \delta\right)=$ $\left\{\omega:\left\|\omega-\omega_{j}\right\|_{m} \leqslant \delta\right\}$ (not proper balls in $\Omega$ ). We have to estimate the differences

$$
\begin{aligned}
\sup _{\omega \in K}\left|\widetilde{G}_{\varepsilon}^{S}(\omega)-G(\omega)\right| \leqslant & \max _{j \in J_{\delta}}\left\{\sup _{\omega \in B_{m}\left(\omega_{j}, \delta\right) \cap K}\left|\widetilde{G}_{\varepsilon}^{S}(\omega)-\widetilde{G}_{\varepsilon}^{S}\left(\omega_{j}\right)\right|+\left|\widetilde{G}_{\varepsilon}^{S}\left(\omega_{j}\right)-G\left(\omega_{j}\right)\right|\right. \\
& \left.+\sup _{\omega \in B_{m}\left(\omega_{j}, \delta\right) \cap K}\left|G\left(\omega_{j}\right)-G(\omega)\right|\right\}
\end{aligned}
$$

and

$$
\begin{aligned}
\sup _{\omega \in K}\left\|\nabla \cdot \widetilde{G}_{\varepsilon}^{S}(\omega)-\nabla \cdot G(\omega)\right\| \leqslant & \max _{j \in J_{\delta}}\left\{\sup _{\omega \in B_{m}\left(\omega_{j}, \delta\right) \cap K}\left\|\nabla \cdot \widetilde{G}_{\varepsilon}^{S}(\omega)-\nabla \cdot G\left(\omega_{j}\right)\right\|+\left\|\nabla \cdot \widetilde{G}_{\varepsilon}^{S}\left(\omega_{j}\right)-\nabla \cdot G\left(\omega_{j}\right)\right\|\right. \\
& \left.+\sup _{\omega \in B_{m}\left(\omega_{j}, \delta\right) \cap K}\left\|\nabla \cdot G\left(\omega_{j}\right)-\nabla \cdot G(\omega)\right\|\right\}
\end{aligned}
$$

where we always choose to place $\omega$ in the ball $B_{m}\left(\omega_{j}, \delta\right)$ for which the center is the closest element from $\omega$ among the finite collection of points $\omega_{j} \in J_{\varepsilon}$. The uniform continuity on $K$ guarantees that the third terms on the right-hand side of both inequalities are of order $\varepsilon$. To estimate the differences $\left|\widetilde{G}_{\varepsilon}^{S}\left(\omega_{j}\right)-G\left(\omega_{j}\right)\right|$ and $\left\|\nabla . \widetilde{G}_{\varepsilon}^{S}\left(\omega_{j}\right)-\nabla \cdot G\left(\omega_{j}\right)\right\|$ we need to look at the values of $g_{\varepsilon}(\bar{x})$ and $\nabla g_{\varepsilon}(\bar{x})$ in a neighborhood $K_{\delta, j^{\prime}}^{x}, j^{\prime} \in J_{\varepsilon}$, and compare to the values at $\omega_{j}$. The values of $g_{\varepsilon} S\left(\omega_{j}\right)$ are the result of convolution with $\phi_{m, \rho}$ of values of $g_{\varepsilon}$ for some $\bar{x}$ with $\left\|\omega_{\bar{x}}-\omega_{j}\right\|_{m}<\rho$. The worst case scenario is that the value of $g_{\varepsilon}$ at $\bar{x}$ is a value of $g_{m, j^{\prime}}$ on an adjacent ball $K_{\delta, j^{\prime}}^{x}$ to $K_{\delta, j}^{x}$ (this fact grants that $\left.\left\|\omega_{j}-\omega_{j^{\prime}}\right\|_{m}<2 \delta<3 \delta\right)$ and then $\left|\widetilde{G}_{\varepsilon}^{S}\left(\omega_{j}\right)-G\left(\omega_{j}\right)\right| \leqslant\left|g_{m, j^{\prime}}(\bar{x})-G\left(\omega_{j^{\prime}}\right)\right|+\left|G\left(\omega_{j^{\prime}}\right)-G\left(\omega_{j}\right)\right|$ which is of order $\varepsilon$ by construction. The same is valid for $\left\|\nabla . \widetilde{G}_{\varepsilon}^{S}\left(\omega_{j}\right)-\nabla . G\left(\omega_{j}\right)\right\|$, bounded above by

$$
\sup _{\bar{x} \in K_{\delta, j^{\prime}}^{x}}\left\|\nabla \cdot g_{m, j^{\prime}}(\bar{x})-\nabla \cdot G\left(\omega_{j^{\prime}}\right)\right\|+\left\|\nabla \cdot G\left(\omega_{j^{\prime}}\right)-\nabla \cdot G\left(\omega_{j}\right)\right\|,
$$


of order $\varepsilon$ as well.

We need to estimate the first terms on the right-hand side of the inequalities. $\left|\widetilde{G}_{\varepsilon}^{S}(\omega)-\widetilde{G}_{\varepsilon}^{S}\left(\omega_{j}\right)\right|$ and $\left\|\nabla \cdot \widetilde{G}_{\varepsilon}^{S}(\omega)-\nabla \cdot \widetilde{G}_{\varepsilon}^{S}\left(\omega_{j}\right)\right\|$ are bounded above by the supremum value of the differences $\left|g_{\varepsilon}\left(\bar{x}^{\prime}\right)-g_{\varepsilon}\left(\bar{x}^{\prime \prime}\right)\right|$ and $\left\|\nabla \cdot g_{\varepsilon}\left(\bar{x}^{\prime}\right)-\nabla \cdot g_{\varepsilon}\left(\bar{x}^{\prime \prime}\right)\right\|$, respectively, where $\left\|\omega_{\bar{x}^{\prime}}-\omega\right\|_{m} \leqslant \rho$ and $\left\|\omega_{\bar{x}^{\prime \prime}}-\omega_{j}\right\|_{m} \leqslant \rho$. Assume the value at $\bar{x}^{\prime}$ is given by $g_{m, j^{\prime}}$ on a ball $K_{\delta, j^{\prime}}^{x}$ and the value at $\bar{x}^{\prime \prime}$ is given by $g_{m, j^{\prime \prime}}$ on a ball $K_{\delta, j^{\prime \prime}}^{x}$. At this point we intercalate the values of the functions at $\omega_{j^{\prime}}, \omega_{j}$ and $\omega_{j^{\prime \prime}}$. The differences between values in the same domain $K_{\delta, l}^{x}$, for any $l \in J_{\varepsilon}$ are of order $\varepsilon$. We only have to compare the values at $\omega_{j^{\prime}}$ and $\omega_{j^{\prime \prime}}$ with $\omega_{j}$. The distance between $\omega_{j^{\prime \prime}}$ and $\omega_{j}$ is less than $\delta+\rho$. First,

$$
\left\|\omega_{j^{\prime \prime}}-\omega_{j}\right\|_{m} \leqslant \sup _{\bar{x} \in K_{\delta, j^{\prime \prime}}^{x}}\left\|\omega_{j^{\prime \prime}}-\omega_{\bar{x}^{\prime \prime}}\right\|_{m}+\left\|\omega_{\bar{x}^{\prime \prime}}-\omega_{j}\right\|_{m} \leqslant \delta+\rho<3 \delta
$$

which implies that the error is of order $\varepsilon$. We know that $\omega_{j}$ is the closest of all $\omega_{l}$, with $l \in J_{\varepsilon}$ from $\omega$. Hence $\left\|\omega-\omega_{j}\right\|_{m} \leqslant\left\|\omega-\omega_{j^{\prime}}\right\|_{m}$. In the same time $\left\|\omega-\omega_{\bar{x}^{\prime}}\right\|_{m} \leqslant \rho$ and $\left\|\omega_{\bar{x}^{\prime}}-\omega_{j^{\prime}}\right\|_{m} \leqslant \delta$. We conclude that $\left\|\omega-\omega_{j^{\prime}}\right\|_{m} \leqslant \delta+\rho$ which implies that $\left\|\omega_{j^{\prime}}-\omega_{j}\right\|_{m} \leqslant 2 \delta+2 \rho<3 \delta$. The difference will be of order $\varepsilon$ once again. We obtained a function $G_{\varepsilon}^{S}(\omega)=g_{\varepsilon}^{S}\left(\omega\left(t_{0}\right), \ldots, \omega\left(t_{m}\right)\right)$ where $g_{\varepsilon}^{S}(\bar{x}) \in C_{0}^{\infty}\left(\mathbb{R}^{m+1}\right)$ within distance $C \varepsilon$ from $G$ inside the compact $K$ in the uniform norm, where $C$ is independent from $G$ and $K$, and bounded by $2\|G\|_{C_{b}^{1}}$. The number $m$ depends on $\varepsilon$ and the compact $K$.

We still have to prove that we can approximate $\widetilde{G}_{\varepsilon}^{S}$ with a function $G \in \mathcal{S}(\Omega)$. For a given $\varepsilon$ and a compact $K$, the function $g_{\varepsilon}^{S}(\bar{x})$ and $m$ are fixed. The function $g_{\varepsilon}^{S}$ has support included in the compact $[-M-1, M+1]^{m+1} \subseteq$ $(-M-3, M+3)^{m+1} \subseteq \mathbb{R}^{m+1}$. Let $K^{\prime}=[-M-2, M+2]^{m+1}$. It is known (for example from [10]) that for any $r \in \mathbb{Z}_{+}$, a function $f \in C^{r}\left(\mathbb{R}^{m+1}\right)$ and all its derivatives can be approximated uniformly on any compact with polynomials in $\mathbb{R}^{m+1}$. The class of polynomials is of cylinder type, in the sense that it is the linear span of products of functions (in this case, polynomials of one variable) of the variables $x_{0}, x_{1}, \ldots, x_{m}$. The problem is that these functions are not of Schwartz class. The indicator function of the compact $K^{\prime}$ is the product of the indicator functions of the interval $[-M-2, M+2]$, hence of cylinder type. The product of the indicator functions with each polynomial will be of cylinder type. We can consider the convolution with $\phi_{m, \rho}(\bar{x})$, with $\rho<\frac{1}{2}$. Let $\varepsilon^{\prime}$ be the accuracy of the approximation in the supremum norm. The function $\phi_{m, \rho}(\bar{x})$ is of cylinder type as well. A consequence of this will be that the result of the convolution will be of class $C_{0}^{\infty}\left(\mathbb{R}^{m+1}\right)$, will be of cylinder type (as the convolution of two cylinder type functions, that is for which the variables decouple in the convolution integral), and will stay within distance $\varepsilon^{\prime}$ uniformly together with all the derivatives.

We choose $\varepsilon^{\prime}=\varepsilon /(m+1)$ and let $h_{\varepsilon^{\prime}}(\bar{x})$ be the sum of cylinder functions with compact support approximating $g_{\varepsilon}^{S}(\bar{x})$. The function $G_{\varepsilon}^{S}(\omega)=h_{\varepsilon^{\prime}}\left(\omega\left(t_{0}\right), \ldots, \omega\left(t_{m}\right)\right)$ is in $\mathcal{S}(\Omega)$ and $\left|G_{\varepsilon}^{S}(\omega)-\widetilde{G}_{\varepsilon}^{S}(\omega)\right| \leqslant \varepsilon^{\prime}<\varepsilon$. We conclude the proof by noticing that

$$
\left\|\nabla \cdot G_{\varepsilon}^{S}(\omega)-\nabla \cdot \widetilde{G}_{\varepsilon}^{S}(\omega)\right\| \leqslant \sum_{0 \leqslant i \leqslant m} \sup _{\bar{x} \in \mathbb{R}^{m+1}}\left\|\partial_{x_{i}} h_{\varepsilon^{\prime}}(\bar{x})-\nabla_{x_{i}} g_{\varepsilon}^{S}(\bar{x})\right\| \leqslant(m+1) \varepsilon^{\prime}=\varepsilon .
$$

Proof of Lemma 1. We can re-write the matrix $V^{N}=\left(V_{i j}^{N}\right)$ as

$$
V_{i i}^{N}=\sigma^{2}+\frac{c_{i}^{N}(\sigma)}{\sqrt{N}} \quad \text { with } \max _{1 \leqslant i \leqslant N}\left|c_{i}^{N}(\sigma)\right| \leqslant c^{N}(\sigma)
$$

and

$$
V_{i j}^{N}=\frac{\gamma \sigma^{2}}{N}+\frac{c_{i j}^{N}(\gamma)}{N} \quad \text { with } \max _{1 \leqslant i, j \leqslant N}\left|c_{i j}^{N}(\gamma)\right| \leqslant c^{N}(\gamma)
$$

where $\lim _{N \rightarrow \infty}\left(c^{N}(\sigma)+c^{N}(\gamma)\right)=0$.

We first prove the positive definiteness. Let $\bar{x}=\left(x_{1}, x_{2}, \ldots, x_{N}\right)$ be an arbitrary $N$-dimensional vector. If $S=\sum_{1 \leqslant i \leqslant N} x_{i}$ 


$$
\begin{aligned}
\left(\bar{x}, V^{N} \bar{x}\right)= & \sum_{1 \leqslant i, j \leqslant N} V_{i j}^{N} x_{i} x_{j}=\sum_{1 \leqslant i \leqslant N}\left(V_{i i} x_{i}+\sum_{j \neq i} V_{i j} x_{j}\right) x_{i} \\
= & \sum_{1 \leqslant i \leqslant N}\left(\sigma^{2}+\frac{c_{i}^{N}(\sigma)}{\sqrt{N}}\right) x_{i}^{2}+\sum_{1 \leqslant i, j \leqslant N, j \neq i}\left(\frac{\gamma \sigma^{2}}{N}+\frac{c_{i j}^{N}(\gamma)}{N}\right) x_{j} x_{i} \\
= & \sigma^{2}\left(\sum_{1 \leqslant i \leqslant N} x_{i}^{2}+\frac{\gamma}{N}\left(S-x_{i}\right) x_{i}\right) \\
& +\sum_{1 \leqslant i \leqslant N} \frac{c_{i}^{N}(\sigma)}{\sqrt{N}} x_{i}^{2}+\sum_{1 \leqslant i, j \leqslant N, i \neq j} \frac{c_{i j}^{N}(\gamma)}{N} x_{j} x_{i} .
\end{aligned}
$$

We can bound (A.15) in absolute norm by

$$
\|\bar{x}\|^{2}\left[\left(\frac{c^{N}(\sigma)}{\sqrt{N}}+\frac{c^{N}(\gamma)}{N}\right)+c^{N}(\gamma)\left(\frac{\left(\sum_{1 \leqslant i \leqslant N}\left|x_{i}\right|\right)^{2}}{N\|\bar{x}\|^{2}}\right)\right]
$$

while this can be bounded above by Schwarz's inequality

$$
\|\bar{x}\|^{2}\left[\frac{c^{N}(\sigma)}{\sqrt{N}}+c^{N}(\gamma)\left(1+\frac{1}{N}\right)\right] \sim \mathrm{o}(1) .
$$

The main term (A.14) is zero if $\|x\|=0$, but otherwise it is equal to

$$
\sigma^{2}\left(1-\frac{\gamma}{N}\right)\|\bar{x}\|^{2}+\frac{\gamma \sigma^{2}}{N} S^{2}=\sigma^{2}\|\bar{x}\|^{2}\left(\left(1-\frac{\gamma}{N}\right)+\gamma \frac{S^{2}}{N\|\bar{x}\|^{2}}\right) .
$$

If $\gamma \geqslant 0$ a lower bound is $\sigma^{2}\|\bar{x}\|^{2}\left(1-\frac{\gamma}{N}\right)$ of order $\mathrm{O}(1)$ and if $\gamma<0$ we can use Schwarz's inequality again and obtain the lower bound $\sigma^{2}\|\bar{x}\|^{2}\left(1+\gamma-\frac{\gamma}{N}\right)$. This proves that $\left(\bar{x}, V^{N} \bar{x}\right) \geqslant \sigma^{2}\|\bar{x}\|^{2} C^{\prime}$ with $C^{\prime}>0$ independent of $N$. The only condition needed to ensure a lower bound uniformly in $N$ is $\gamma>-1$.

We proceed to the proof of the existence of $\left\{w_{i}^{N}\right\}_{1 \leqslant i \leqslant N}$. We first write $\left\{v_{i}^{N}\right\}_{1 \leqslant i \leqslant N}$ in an orthonormal basis $\left\{e_{i}\right\}_{1 \leqslant i \leqslant N}$ with the matrix $R=\left(r_{k l}\right)$ such that $v_{i}=\sum_{1 \leqslant k \leqslant N} r_{i k} e_{k}$. With this notation $V=R R^{*}$ and since $R R^{*}=(\sqrt{V})^{2}$ and we have already shown that $V$ is positive definite, there exists a unitary matrix $U=\left(u_{k l}\right)$ defined directly by $U=(\sqrt{V})^{-1} R$. We write $R=\sqrt{V} U$. Let

$$
w_{i}=\sigma \sum_{1 \leqslant k \leqslant N} u_{i k} e_{k} .
$$

We have to show that the elements of the diagonal of $(R-\sigma U)(R-\sigma U)^{*}$ are uniformly bounded by a constant of order $\mathrm{O}\left(N^{-1}\right)$. Since $(R-\sigma U)$ can be written as $\sigma\left(\sigma^{-1} \sqrt{V}-I\right) U$ we have

$$
\left|\left(\left(\sigma^{-1} \sqrt{V}-I\right) U U^{*}\left(\sigma^{-1} \sqrt{V}-I\right) e_{i}, e_{i}\right)\right|=\left\|\left(\sigma^{-1} \sqrt{V}-I\right) e_{i}\right\|^{2} \leqslant\left\|\left(\sigma^{-2} V-I\right) e_{i}\right\|^{2}
$$

by contraction. To see this, we denote $y_{i}=\left(\sigma^{-1} \sqrt{V}-I\right) e_{i}$ for all $1 \leqslant i \leqslant N$ and $A=\sigma^{-1} \sqrt{V}+I$. The positive definiteness of $V$ implies that $A^{-1}$ is a contraction. If $\left(\delta_{i j}\right)$ denotes the unit matrix, the bound for the diagonal term of $R-\sigma U$ is

$$
\left\|\left(V-\sigma^{2} I\right) e_{i}\right\|^{2}=\sum_{1 \leqslant k \leqslant N}\left(V_{i k}-\sigma^{2} \delta_{i k}\right)^{2} .
$$

We recall that the diagonal term is of order $\mathrm{O}\left(N^{-1 / 2}\right)$ and all the non-diagonal terms are of order $\mathrm{O}\left(N^{-1}\right)$, uniformly over the set of indices $1 \leqslant i, j \leqslant N$. This concludes the proof. 


\section{References}

[1] V.I. Bogachev, Gaussian Measures, in: Mathematical Surveys and Monographs, vol. 62, American Mathematical Society, Providence, RI, 1998.

[2] C.C. Chang, H.-T. Yau, Fluctuations of one-dimensional Ginzburg-Landau models in nonequilibrium, Comm. Math. Phys. 145 (1992) 209-234.

[3] N. Dunford, J. Schwartz, Linear Operators, Part I, General Theory, Wiley, 1988.

[4] G. Gielis, A. Koukkous, C. Landim, Equilibrium fluctuations for zero range processes in random environment, Stochastic Process. Appl. 77 (2) (1998) 187-205.

[5] I. Grigorescu, Self-diffusion for Brownian motions with local interaction, Ann. Probab. 27 (3) (1999) $1208-1267$.

[6] I. Grigorescu, Large scale behavior of a system of interacting diffusions, in: Hydrodynamic Limits and Related Topics (Toronto, ON, 1998), in: Fields Inst. Commun., vol. 27, Amer. Math. Society, Providence, RI, 2000, pp. 83-93.

[7] R. Holley, D.W. Stroock, Central limit phenomena of various interacting systems, Ann. of Math. (2) 110 (2) (1979) $333-393$.

[8] N. Ikeda, S. Watanabe, Stochastic Differential Equations and Diffusion Processes, Second edition, North-Holland/Kodansha, 1989.

[9] K. Itô, Distribution-valued processes arising from independent Brownian motions, Math. Z. 182 (1) (1983) 17-33.

[10] F. John, Partial Differential Equations, Fourth edition, in: Applied Mathematical Sciences, vol. 1, Springer-Verlag, New York, 1991.

[11] C. Kipnis, C. Landim, Scaling Limits of Interacting Particle Systems, Springer-Verlag, New York, 1999.

[12] J. Quastel, Diffusion of color in the simple exclusion process, Comm. Pure Appl. Math. 45 (1998) 321-379.

[13] J. Quastel, F. Rezakhanlou, S.R.S. Varadhan, Large deviations for the symmetric simple exclusion process in dimensions $d \geqslant 3$, Probab. Theory Related Fields 113 (1) (1999) 1-84.

[14] K. Ravishankar, Fluctuations from the hydrodynamical limit for the symmetric simple exclusion in $Z^{d}$, Stochastic Process. Appl. 42 (1) (1992) 31-37.

[15] A.N. Shiryaev, Probability, Translated from the Russian by R.P. Boas, in: Graduate Texts in Math., vol. 95, Springer-Verlag, New York, 1984.

[16] A.S. Sznitman, A fluctuation result for nonlinear diffusions, in: Infinite-Dimensional Analysis and Stochastic Processes (Bielefeld, 1983), in: Res. Notes in Math., vol. 124, Pitman, Boston, 1985, pp. 145-160. 